MIT-CTP-3988

SLAC-PUB-13388

December 2008

\title{
Supersymmetry Without Prejudice *
}

\author{
Carola F. Berger ${ }^{\dagger}$ \\ Center for Theoretical Physics, Massachusetts Institute of Technology, Cambridge, MA 02139, USA \\ James S. Gainer ${ }^{\ddagger}$, JoAnne L. Hewett ${ }^{\S}$, Thomas G. Rizzo $₫$ \\ SLAC National Accelerator Laboratory, 2575 Sand Hill Rd., Menlo Park, CA, 94025, USA
}

\begin{abstract}
We begin an exploration of the physics associated with the general CP-conserving MSSM with Minimal Flavor Violation, the pMSSM. The 19 soft SUSY breaking parameters in this scenario are chosen so as to satisfy all existing experimental and theoretical constraints assuming that the WIMP is a thermal relic, i.e., the lightest neutralino. We scan this parameter space twice using both flat and log priors for the soft SUSY breaking mass parameters and compare the results which yield similar conclusions. Detailed constraints from both LEP and the Tevatron searches play a particularly important role in obtaining our final model samples. We find that the pMSSM leads to a much broader set of predictions for the properties of the SUSY partners as well as for a number of experimental observables than those found in any of the conventional SUSY breaking scenarios such as mSUGRA. This set of models can easily lead to atypical expectations for SUSY signals at the LHC.
\end{abstract}

\footnotetext{
*Work supported in part by the Department of Energy, Contract DE-AC02-76SF00515

${ }^{\dagger}$ e-mail:cfberger@mit.edu

‡e-mail:jgainer@slac.stanford.edu

$\S$ e-mail:hewett@slac.stanford.edu

ฯe-mail:rizzo@slac.stanford.edu
}

Published in the Journal of High Energy Physics (JHEP) 


\section{Introduction}

The LHC will soon begin operations in earnest and will thereafter begin a detailed study of the Terascale. Amongst the many prospective new physics signals that may be realized at the LHC, the possible observation of an excess of events with missing energy is awaited with great anticipation. Given the strong collection of evidence for the existence of dark matter [1] and the naturalness of the WIMP paradigm [2], it is no surprise that many beyond the Standard Model (SM) scenarios predict the appearance of missing energy signatures at the LHC [3]. Perhaps the most well explored of these scenarios is R-Parity conserving, N=1 Supersymmetry (SUSY) where the WIMP is identified as the lightest supersymmetric particle (LSP), which most commonly corresponds to the lightest neutralino or gravitino. The simplest manifestation of this picture is the Minimal Supersymmetric Standard Model (MSSM), where the gauge group of the SM is maintained and the matter content of the SM is augmented only by an additional Higgs doublet.

While the MSSM has many nice features, perhaps its least understood aspect is the nature of the mechanism which breaks the Supersymmetry. There is an ever growing list of possible candidate scenarios, including mSUGRA [4], GMSB [5], AMSB [6], and gaugino mediated supersymmetry breaking [7]. Each of these mechanisms strongly influences both the mass spectra and decay patterns of the various sparticles and hence it is highly non-trivial to make model-independent statements about the properties of the LSP or about SUSY signatures at colliders such as the LHC. This puts forth the question of whether there is some way to study the MSSM in a broad fashion while simultaneously adopting as few simplifying assumptions as possible. The purpose of this paper is to address this issue. In trying to answer this question, one is immediately faced with the daunting task of dealing with the over 120 parameters, arising mainly in the soft-breaking sector, which describe SUSY breaking in the MSSM [8]. Clearly the phenomenological study of any model with such a large number of parameters is totally hopeless. Obviously some assumptions need to be made in order to reduce this large number of independent parameters to a viable subset and there are many possible avenues one could follow. Most studies assume a specific SUSY breaking 
mechanism, employing theoretical assumptions at the GUT scale, and thus reduce the number of independent model parameters to 4 or 5 . In this investigation, we will not make any assumptions at the high scale. We will, however, restrict ourselves to the CP-conserving MSSM (i.e., no new phases) with minimal flavor violation (MFV)[9]. Moreover, to simplify matters further and help soften the impact of the experimental constraints arising from the flavor sector, we will require that the first two generations of sfermions be degenerate. This leaves us with 19 independent, real, weak-scale, SUSY Lagrangian parameters to consider. These include the gaugino masses $M_{1,2,3}$, the Higgsino mixing parameter $\mu$, the ratio of the Higgs vevs $\tan \beta$, the mass of the pseudoscalar Higgs boson $m_{A}$, and the 10 squared masses of the sfermions ( 5 for the assumed degenerate first two generations and a separate 5 for the third generation). Finally, due to the small Yukawa couplings for the first two generations, independent $A$-terms are only phenomenologically relevant for the third generation and will be included in our study for the $b, t$ and $\tau$ sectors. This set of 19 parameters leaves us with what has been called the phenomenological MSSM (pMSSM) in the literature[10] and is the scenario that we will study in some detail below.

The primary goal of the first phase of this study is to obtain a large set of MSSM parameter points that can be used for future studies of SUSY signatures, e.g., signals at the LHC or in cosmic rays. To accomplish this, we will perform a scan over this 19-dimensional parameter space and subject the resulting specific sets of parameter choices (which we denote as models) to a large and well-known (and some perhaps, not so well-known) set of experimental and theoretical constraints[11]. We will do this with some care to identify sets of parameter space points which are at present phenomenologically viable. As we will see below, applying these constraints can be complicated and is not always as straightforward as employed in some other analyses. This is particularly true in the case where experimental bounds are predicated on specific SUSY breaking mechanisms. As we are not a priori interested in any particular region of parameter space (e.g., we are enforcing the WMAP dark matter density[12] observations only as an upper bound) we will not need to make use of Markov-chain Monte Carlo techniques[13, 14]. We will then perform a detailed 
study of the properties of these surviving models and, in particular, highlight those aspects which are not generally encountered when only a given set of SUSY breaking scenarios is considered. This allows us to get a perspective on the wide range of predictions for various observables that can arise in the more general pMSSM framework in comparison to those found in specific SUSY breaking scenarios.

In the next Section, we will discuss our scanning techniques, the ranges of the soft breaking parameters that we employ, as well as the values of the SM input parameters that we use. In Section 3, we will detail the various theoretical and experimental constraints at some length and will explain exactly how they are employed in our analysis. Section 4 presents our analysis and a survey of the results, while Section 5 contains an overview discussion of the lessons learned from this analysis. Our conclusions can be found in Section 6 .

\section{Parameters Values and Ranges}

To perform our multi-dimensional parameter scan, we need to choose suitable ranges for the softbreaking parameters that we employ and determine how the values of these parameters are picked within these assumed ranges. In the analysis presented here, we will make two independent pa-

rameter scans. In the first case, we randomly generate $10^{7}$ sets of parameters, assuming flat priors, i.e., we assume that the parameter values are chosen uniformly throughout their allowed ranges. With an eye toward the experimental constraints to be discussed in the next Section, for the flat 
prior scan we employ the following ranges:

$$
\begin{array}{r}
100 \mathrm{GeV} \leq m_{\tilde{f}} \leq 1 \mathrm{TeV}, \\
50 \mathrm{GeV} \leq\left|M_{1,2}, \mu\right| \leq 1 \mathrm{TeV}, \\
100 \mathrm{GeV} \leq M_{3} \leq 1 \mathrm{TeV}, \\
\left|A_{b, t, \tau}\right| \leq 1 \mathrm{TeV}, \\
1 \leq \tan \beta \leq 50, \\
43.5 \mathrm{GeV} \leq m_{A} \leq 1 \mathrm{TeV} .
\end{array}
$$

Note that absolute value signs are present in these quoted ranges as we will allow the soft-breaking parameters to have arbitrary sign. Given that the soft parameters $M_{i}, A_{i}$ and $\mu$ can all appear with arbitrary signs (i.e., phases) but that only six relative signs are physical[15] we choose $M_{3}$ to be positive. This scan will generate SUSY spartners with light to moderate masses.

For our second scan, we randomly generate $2 \times 10^{6}$ sets of parameters assuming log priors for the mass parameters. We then modify the allowed soft parameter ranges as follows:

$$
\begin{array}{r}
100 \mathrm{GeV} \leq m_{\tilde{f}} \leq 3 \mathrm{TeV}, \\
10 \mathrm{GeV} \leq\left|M_{1,2}, \mu\right| \leq 3 \mathrm{TeV}, \\
100 \mathrm{GeV} \leq M_{3} \leq 3 \mathrm{TeV}, \\
10 \mathrm{GeV} \leq\left|A_{b, t, \tau}\right| \leq 3 \mathrm{TeV}, \\
1 \leq \tan \beta \leq 60, \\
43.5 \mathrm{GeV} \leq m_{A} \leq 3 \mathrm{TeV} .
\end{array}
$$

Here, $\tilde{f}=\tilde{Q}_{L}, \tilde{Q}_{3}, \tilde{L}_{1}, \tilde{L}_{3}, \tilde{u}_{1}, \tilde{d}_{1}, \tilde{u}_{3}, \tilde{d}_{3}, \tilde{e}_{1}$, and $\tilde{e}_{3}$. This expanded range of parameters will allow us access to both very light as well as some heavy sparticle states that may only be produced at the SLHC. The goal of this second scan is to make contrasts and comparisons to the flat prior study in order to determine the dependence of the model properties on the scan assumptions. 
Note that the above mass parameters are assumed to be evaluated at the SUSY scale, i.e., by convention the geometric mean of the two stop masses $\lesssim 1 \mathrm{TeV}$. The physical spectra for the sparticles themselves are generated in all cases using the code SuSpect2.34[10]. Once a set of parameters is chosen, we subject the resulting MSSM model to the entire set of constraints discussed in the next Section; those models which survive will be analyzed in further detail below. Of course, as models are passed through each successive experimental 'filter,' the number of surviving models will be further and further reduced.

Another important aspect of such a broad study is the set of input values for the SM parameters, e.g., $\alpha_{s}\left(M_{Z}\right), m_{t}, M_{W}$, etc., that we employ. As is well-known, some of the predictions for the various observables we analyze below can be sensitive to these particular numerical choices. While we have chosen these numerical values with some care, we are not advocating any one particular set of values for these parameters. We provide their values here only for completeness since it is important for the interested reader to know exactly how our analysis was performed. Table 1 lists the values of these SM parameters used in our analysis. While we only quote central values here, many of the relevant errors are included when evaluating the set of observables we discuss in the next Section. We follow this approach, rather than including the SM input values in our scan, since they are essentially known quantities unlike the pMSSM parameters.

\section{Theoretical and Experimental Constraints}

Apart from the restrictions imposed by the ranges chosen above for the 19 pMSSM soft breaking parameters, there are a large number of both theoretical and experimental constraints that need to be considered in order to obtain phenomenologically viable models. Many of these are familiar

while others will be somewhat less so to the arbitrary reader. The direct application of subsets of these numerous constraints and the specific ranges which are considered viable varies widely in the literature from one analysis to another[22]; hence, we will discuss them here in some in detail. 


\begin{tabular}{|c|c|}
\hline Parameter & Value \\
\hline$\alpha\left(M_{Z}\right)$ & $127.918 \quad$ [Ref.[16]] \\
\hline$\alpha_{s}\left(M_{Z}\right)$ & $0.1198 \quad$ [Ref.[17]] \\
\hline$M_{Z}$ & 91.1875 GeV [Ref.[18]] \\
\hline$\Gamma_{Z}$ & $2.4952 \mathrm{GeV} \quad[\operatorname{Ref} .[16,18]]$ \\
\hline$\left.\sin ^{2} \theta_{w}\right|_{\text {on-shell }}$ & $0.22264 \quad$ [Ref.[18]] \\
\hline$M_{W}$ & $80.398 \mathrm{GeV} \quad$ [Ref.[18]] \\
\hline$\Gamma_{W}$ & $2.140 \mathrm{GeV} \quad$ [Ref.[18]] \\
\hline$m_{s}(1 \mathrm{GeV})$ & $128 \mathrm{MeV} \quad$ [Ref.[16]] \\
\hline$m_{c}^{\text {pole }}$ & $1666 \mathrm{GeV}$ [Ref.[17]] \\
\hline$m_{b}$ & $4.164 \mathrm{GeV} \quad$ [Ref.[17]] \\
\hline$m_{b}^{\text {pole }}$ & $4.80 \mathrm{GeV} \quad[\operatorname{Ref} .[17]]$ \\
\hline$m_{t}^{\text {pole }}$ & $172.6 \mathrm{GeV} \quad$ [Ref.[19]] \\
\hline$V_{u s}$ & 0.2255 [Ref.[16]] \\
\hline$V_{c b}$ & $41.6 \times 10^{-3}[$ Ref. $[16]]$ \\
\hline$V_{u b}$ & $4.31 \times 10^{-3}[$ Ref. $[16]]$ \\
\hline$V_{u b} / V_{c b}$ & $0.104 \quad$ [Ref.[16]] \\
\hline$m_{B_{d}}$ & $5.279 \mathrm{GeV} \quad$ [Ref.[16]] \\
\hline$f_{B_{d}}$ & $216 \mathrm{MeV} \quad$ [Ref.[16]] \\
\hline$\tau_{B_{d}}$ & $1.643 \mathrm{ps} \quad$ [Ref.[16]] \\
\hline$f_{B_{s}}$ & $230 \mathrm{MeV} \quad$ [Ref.[20]] \\
\hline$\tau_{B_{S}}$ & $1.47 \mathrm{ps} \quad[$ Ref.[21]] \\
\hline
\end{tabular}

Table 1: Central values of the SM input parameters used in our analysis. 
The first set of constraints we impose are theoretical and rather standard and are applied while generating the sparticle spectrum with the SuSpect code: the sparticle spectrum must be tachyon free and cannot lead to color or charge breaking minima in the scalar potential. We also require that electroweak symmetry breaking be consistent and that the Higgs potential be bounded from below. Note, however, since we do not impose grand unification (as we are ignorant of the ultraviolet completion of the MSSM if we don't implement a specific SUSY breaking scenario), we do not require that the RGE evolution of the couplings be well-behaved up to the GUT scale. Because we are only concerned with the physics between the weak and $\mathrm{TeV}$ scales and the overall effects of RGE evolution in this range will be rather modest, it is unlikely that a Landau pole or other irregularities can be generated in this narrow mass window.

At this point in our analysis, we will make the further assumption that the WIMP be a thermal relic so that the LSP can be identified as the lightest neutralino; the possibility of the LSP being the gravitino or the axino will be ignored in the rest of the present analysis[23]. The scenario where the gravitino is the LSP[24] will be discussed in a future analysis within a different astrophysical framework. We note that the case where the sneutrino is the LSP can be easily eliminated in the pMSSM by combining several of the constraints discussed below.

The code micrOMEGAs2.21[25] takes the MSSM spectrum output from SuSpect and allows us to implement the constraints arising from a number of precision and flavor measurements: precision electroweak constraints via $\Delta \rho$, the rare decays $b \rightarrow s \gamma$ and $B_{s} \rightarrow \mu^{+} \mu^{-}$, as well as the $g-2$ of the muon. We take the $95 \%$ CL allowed experimental range for $\Delta \rho$ directly from the analysis presented in the $2008 \mathrm{PDG}[16]:-0.0007 \leq \Delta \rho \leq 0.0026$. For $b \rightarrow s \gamma$ we use the combined experimental result from HFAG[21], $B_{b \rightarrow s \gamma}=(3.52 \pm 0.25) \cdot 10^{-4}$, as well as the recent SM theoretical predictions as given by both Misiak et al.[26] andBecher and Neubert[27]. Combining both the experimental and theoretical errors, we require, rather conservatively, that the predicted branching fraction lie in the range $B_{b \rightarrow s \gamma}=(2.5-4.1) \cdot 10^{-4}$. For the decay $B_{s} \rightarrow \mu^{+} \mu^{-}$, we employ the recently reported combined limit on the branching fraction obtained by CDF and D0[28], 
$B_{B_{s} \rightarrow \mu \mu} \leq 4.5 \cdot 10^{-8}$ at $95 \%$ CL.Note that our philosophy will be to apply some experimental constraints rather loosely, as they can always be tightened in obtaining a final sub-sample. This will also allow us to easily study the sensitivity of the various observables to the particular values of the soft breaking parameters as well as determine the favored ranges of the various observables themselves.

As is well-known, measurements of the anomalous magnetic moment of the muon, $(g-2)_{\mu}$, differ[29] from the recently updated prediction of the SM by more than $\sim 3 \sigma$ : this difference is found to be $\Delta(g-2)_{\mu}=(30.2 \pm 8.8) \cdot 10^{-10}$ in one recent analysis[30] and $(29.5 \pm 7.9) \cdot 10^{-10}$ in another[31], with both analyses using low energy $e^{+} e^{-}$data as input. It has been argued that this deviation might be a signal for new physics beyond the SM, e.g., light Supersymmetry. In order to explore this possibility more fully within the general pMSSM context and also to allow for the scenario that the SM value may essentially end up being correct once the theoretical, experimental and input errors are more fully understood and reduced, we will implement the loose constraint that $\left(-10 \leq \Delta(g-2)_{\mu} \leq 40\right) \cdot 10^{-10}$ in our analysis. This will also allow us to observe how the MSSM predictions are distributed within this range for the set of surviving models.

In addition to these constraints which are essentially built into the micrOMEGAs2.21 code, we employ several others that arise from heavy flavor measurements: $(i)$ the branching fraction for $B \rightarrow \tau \nu$; combining the results from HFAG[21] and those presented at ICHEP08[32] we will require this branching fraction to lie in the range $B_{B \rightarrow \tau \nu}=(55-227) \cdot 10^{-6}$. Here we make use of the theoretical analysis by Isidori and Paradisi[33] as well as Erikson, Mamoudi and Stal[34] which include the SUSY loop contributions to the bottom quark Yukawa coupling. (ii) With the assumption of MFV[9] and degenerate first and second generation sfermions, the resulting constraints from meson-antimeson mixing on the squark mass spectra are relatively modest[35]. We performed a detailed numerical study which demonstrated that these conditions can be generally satisfied if the ratio of first/second and third generation squark soft breaking masses (of a given charge and helicity) differ from unity by no more than a factor of $\sim 5$. We will impose this 
restriction on the mass spectra of the models we consider. We also apply similar restrictions in the slepton sector.

It is well-known that restrictions on the MSSM parameter space arise from employing the LSP as a form of dark matter. Two major constraints are found in this case: the first arises from the 5 year WMAP measurement[12] of the relic density, which we employ only as an upper limit on the LSP contribution, i.e., $\left.\Omega h^{2}\right|_{L S P} \leq 0.1210$. Here we simply acknowledge the possibility that even within the MSSM and the thermal relic framework, dark matter may have multiple components with the LSP being just one possible contributor. The second constraint arises from direct detection searches[36] for dark matter via both spin-independent and spin-dependent LSP elastic scattering off nuclei in the laboratory. Over essentially all of the parameter space, the spin-independent constraints are presently found to be dominant. We include the cross section bounds arising from XENON10[37], CDMS[38], CRESST I[39] and DAMA[40] data. Given the uncertainties from low energy physics (e.g., nuclear form factors) in the determination of these cross sections, we allow for a factor of 4 uncertainty in these calculations. This numerical factor was obtained by varying the low energy input parameters independently over their allowed ranges for several benchmark points. Thus we will allow models to predict cross sections as much as 4 times larger than the usually quoted experimental bounds. We employ micrOMEGAs2.21[25] in evaluating both $\Omega h^{2}$ as well as the dark matter scattering cross sections.

We now turn to the constraints imposed by LEP data. First, we require that there be no new charged sparticles or charged Higgs bosons with masses below $M_{Z} / 2$ due to the lack of evidence for such states in both direct or indirect measurements at the $Z$ pole; we also impose this same constraint on the lightest neutral Higgs boson. Based on data from LEPII[41], we further require that there be no new stable charged particles of any kind with masses below $100 \mathrm{GeV}$. We note thatsuch a situation may occur if, for example, the NLSP is charged and highly degenerate with the LSP, which is a common occurrence in the AMSB scenario. Additionally, we constrain any new contributions to the invisible width of the $Z$ boson to be $\leq 2 \mathrm{MeV}[18]$. This will restrict the masses 
and couplings of light neutralinos that have significant Higgsino content and thus may appear in the decay $Z \rightarrow \chi_{1}^{0} \chi_{1}^{0}$. While it is unlikely that decays such as $Z \rightarrow \chi_{2}^{0} \chi_{1}^{0}$ will be kinematically accessible, in such cases the $\chi_{2}^{0}$ will more than likely decay into visible final states. We isolate such parameter space model points in the analysis below and scrutinize them further ${ }^{\|}$(using the SUSY-HIT1.1[42] to analyze the $\chi_{2}^{0}$ decays). Furthermore, we note that for the range of sfermion mass soft breaking parameters we consider, $Z$ decay to pairs of sneutrinos is not kinematically allowed so that this final state cannot contribute in any way to the purely invisible width.

Data from LEPII provides further direct search constraints on the sparticles, although they are oftentimes sensitive to detailed assumptions about the model; we provide only an outline of these many bounds here. ALEPH[43] has placed a lower limit on the light squark masses, assuming that the gluino is more massive than the squarks, via their decay to a jet+LSP, i.e., jet + missing energy. Provided that the mass difference between the squark and the LSP, $\Delta m$, is $\geq 10 \mathrm{GeV}$ to avoid very soft jets, they obtain a lower limit of $92 \mathrm{GeV}$ on the squark masses. Given our soft breaking parameter spectrum ranges above, we employ this constraint directly, including the $\Delta m$ cut. Models in our scan with $\Delta m<10 \mathrm{GeV}$ are hence not affected by this search limit. A comparable search[44] for light sbottoms using the same sort of decay pattern results in a lower bound of $95 \mathrm{GeV}$ on the mass of this sparticle. Corresponding lower bounds have been placed[45] on the masses of right-handed sleptons undergoing decays to leptons plus missing energy of $m \gtrsim 100(95,90) \mathrm{GeV}$ for the selectron(smuon,stau). This limit is only applicable if the slepton masses are at least a few percent larger than that of the LSP, otherwise the final state leptons will be too soft. As in the case of squark production, this mass splitting requirement is critical and is included in our analysis. Strictly speaking, these bounds are only applicable to the right-handed sleptons, however, they may also be applied to left-handed sleptons provided the corresponding sneutrino $t$-channel exchange contribution is not very important. We make this assumption in our analysis. An analogous situation applies to chargino production. If the LSP-chargino mass

\footnotetext{
"In the one model that falls into this catagory we find that $\chi_{2}^{0}$ is essentially stable but its contribution to the invisible width of the $Z$ is tiny.
} 
splitting is $\Delta m>2 \mathrm{GeV}$, a direct lower limit of $103 \mathrm{GeV}$ on the chargino mass is obtained from LEPII data. However, if this splitting is $\Delta m<2 \mathrm{GeV}$, the bound degrades to $95 \mathrm{GeV}$, provided that also $\Delta m>50 \mathrm{MeV}$, otherwise the chargino would appear as a stable particle in the detector and would then be excluded by the stable particle searches discussed above. In the case where the lightest chargino is dominantly Wino, this limit is found to be applicable only when the electron sneutrino is more massive than $160 \mathrm{GeV}$. There are also two searches for light stops to consider: if $\tilde{t}_{1}$ is too light to decay into $W b \chi_{1}^{0}$, then the search for the decay $\tilde{t}_{1} \rightarrow c \chi_{1}^{0}$ provides a lower bound on the stop mass of $97 \mathrm{GeV}$. If $\tilde{t}_{1}$ is heavy enough to decay into $\ell b \tilde{\nu}$, then the corresponding lower bound is $95 \mathrm{GeV}$.

As provided in detail by the LEP Higgs Working Group[47], there are five sets of constraints on the MSSM Higgs sector imposed by LEPII data. We employ the SUSY-HIT routine to analyze these, recalling that the uncertainty on the calculated mass of the lightest Higgs boson is approximately $3 \mathrm{GeV}[46]$ as determined by SuSpect. The first pair of these constraints applies to the products $g_{Z Z h}^{2} \times B\left(h \rightarrow b \bar{b}, \tau^{+} \tau^{-}\right)$, where $g_{Z Z h}$ represents the $Z Z h$ coupling, relative to their SM values as a function of the light Higgs mass. A second pair of constraints applies to the corresponding couplings $g_{Z h A}^{2} \times B\left(h A \rightarrow b \bar{b} b \bar{b}, b \bar{b} \tau^{+} \tau^{-}\right)$as a function of the sum of the $h$ and $A$ masses. The last of these LEPII Higgs bounds applies to the mass of the charged Higgs boson as a function of its branching fraction into the $\tau \nu$ and $c \bar{s}$ final states. We include all of these experimental constraints in detail in our results presented below.

The final set of constraints we incorporate arises from Tevatron data. We first consider the restrictions imposed on the squark and gluino sectors arising from the null result of the multijet plus missing energy search performed by D0[48]. In our study, we generalize their analysis to render it model independent. For each of our models that have survived the set of constraints thus far, we compute the NLO SUSY cross sections for squark and gluino production using PROSPINO2.0[49]. Once produced, these sparticles are decayed via SDECAY/HDECAY to obtain the relevant decay chains and branching fractions and are then passed to PYTHIA6.4[50] to include hadronization 
and fragmentation. We then use PGS4[51] to simulate the D0 detector and to impose the analysis kinematic cuts; we tune PGS4 by reproducing the results and efficiencies for the three benchmark mSUGRA points chosen by D0 in their published multijet study. Given an integrated luminosity of $2.1 \mathrm{fb}^{-1}$, we find that the $95 \%$ CL upper limit on the number of signal events from combining all of the production channels is 8.34 using the method of Feldman and Cousins[52]. event rate larger than this value, that model is removed from the remaining sample.

Analogously, we employ constraints from the CDF search for trileptons plus missing energy[53], which we also generalize to the full pMSSM. Our procedure is essentially the same as in the jets plus plus missing energy analysis described above, except that we now employ a CDF tuning for PGS4 which we obtain by reproducing the CDF benchmark point results. Here we use the leading order cross section together with a universal K-factor of 1.3 to mimic the full NLO cross section. We used PROSPINO to check that this K-factor is a good approximation for the range of gaugino masses relevant to the Tevatron. In this case we only make use of the ' 3 tight lepton' analysis from CDF as it is the cleanest and easiest to implement with PGS4. The 95\% CL upper bound on the possible SUSY signal in this channel is then 4.65 events assuming a luminosity of $2.02 \mathrm{fb}^{-1}$ as used in the CDF analysis. Again, parameter sets leading to larger event rates are dropped from the remainder of our analysis.

Over the narrow mass range $90 \leq m_{A} \leq 100 \mathrm{GeV}$, searches for SUSY Higgs signals by both CDF and D0[54] provide the approximate constraint $\tan \beta \geq 1.2 m_{A}-70$. We include this restriction in our analysis.

Finally, both CDF[55] and D0[56] have placed lower limits on the masses of heavy stable charged particles. In our analysis we employ the stronger D0 constraint which we take to be of the form $m_{\chi^{+}} \geq 206\left|U_{1 w}\right|^{2}+171\left|U_{1 h}\right|^{2} \mathrm{GeV}$ at $95 \%$ CL in the case of charginos. Here, the matrix $U$ determines the Wino/Higgsino content of the lightest chargino and is used to interpolate between the separate Wino and Higgsino results as quoted by D0. As we will see below, this is an extremely powerful constraint for a large number of our models since we find that chargino-LSP 
mass degeneracies are common in our model sample, particularly when the LSP is nearly pure Wino or Higgsino or a combination of these two cases.

We note in passing that both CDF and D0 also have a set of analyses aimed at searches for light stops and bottoms[57] with various assumptions about the SUSY mass spectrum and possible decay channels. Most of these searches are only applicable if these sparticles are lighter than the top quark. Since these are a difficult set of analyses to generalize to the pMSSM we will constrain our model set to the subset of those which contain stops and sbottoms with masses larger than $m_{t}$. We separately store those models satisfying all of our other constraints above but contain lighter stops and sbottoms (with masses above the LEPII limits) for a further later detailed study. However, models in this category are found to be extremely rare as will be obvious from the stop and sbottom mass distributions presented in the next Section.

\section{Analysis and Results}

We now describe the results from our analysis for both the cases of flat and logarithmic priors.

\subsection{Flat Priors}

In the case of flat priors, we randomly generated $10^{7}$ parameter space points (i.e., models). After subjecting these models to all of the constraints detailed above, we found that only $\sim 68.5 \cdot 10^{3}$ satisfy all of the restrictions. Here, we discuss the properties of these surviving models in some detail. The first characteristic one would like to know is the nature of the sparticle spectra in these various models. Since we cannot analyze so many complete spectra here, we will instead examine a number of specific properties of the models which then will provide an overall qualitative feel for their general behavior.

Figures $1-6$ show histograms of the distributions of the various sparticle masses, as well as for the charged and neutral Higgs boson masses, in our set of surviving models . In Fig. 1, we display 
the distributions of the selectron/smuon, stau and sneutrino masses. We observe several features: (i) right-handed selectrons/smuons tend to be lighter than their corresponding left-handed sleptons, (ii) the lighter stau tends to be the lightest charged slepton, i.e., there is an inclination for it to be lighter than selectrons or smuons with either helicity label; this is most likely due to stau mixing effects, (iii) tau sneutrinos have a proclivity to be lighter than electron/muon sneutrinos (which is not surprising, recalling the connection between the masses of the left-handed charged sleptons and their associated sneutrinos), and (iv) a reasonable fraction of these models, $\gtrsim 10 \%$, would predict that some type of slepton is kinematically accessible at a $500 \mathrm{GeV} e^{+} e^{-}$linear collider.

We present the squark mass distributions in Fig. 2, where we see that the lightest stops and sbottoms are naturally heavier than the top mass (and thus the constraint on stop/sbottom masses imposed above is found to have little effect). The upper left panel reveals particularly interesting results for the first/second generation (i.e., 'light') squarks. Here we see that there are a significant number of models with first/second generation squarks masses below $\sim 300 \mathrm{GeV}$, i.e., well below the limits usually quoted from Tevatron squark searches[48]. It is important to remember that all the models shown here have been subjected to and have passed the Tevatron search constraints from D0. However, the Tevatron searches are targeted at mSUGRA models, which have a very restricted type of mass spectra. There are several ways that light squarks may survive the D0 analysis and not be excluded: $(i)$ if a left-handed squark is light it will tend not to decay immediately to missing energy but instead undergo a cascade (assuming the electroweak gauginos are also light). In this case the D0 search strategy can easily fail as the search criterion are invalid. (ii) If a right-handed squark is relatively light, it will more than likely decay into a jet plus the LSP. However if the mass difference between the squark and the LSP is relatively small (something that does not normally happen in mSUGRA) the resulting events will contain rather soft jets and will not be affected by the kinematic cuts imposed by D0. (iii) Depending on the gluino mass, the production rate of the SUSY signal can be suppressed so that there are not enough events to reach the statistical boundary for an observable signal. This result is important as relatively light squarks could exist 
and may have been missed by the Tevatron searches. In fact, there appears to be a reasonable window for light right-handed squarks to be kinematically accessible at a $500 \mathrm{GeV}$ linear collider. Hunters of squarks at the LHC must keep this possibility in mind when constructing detailed search strategies.
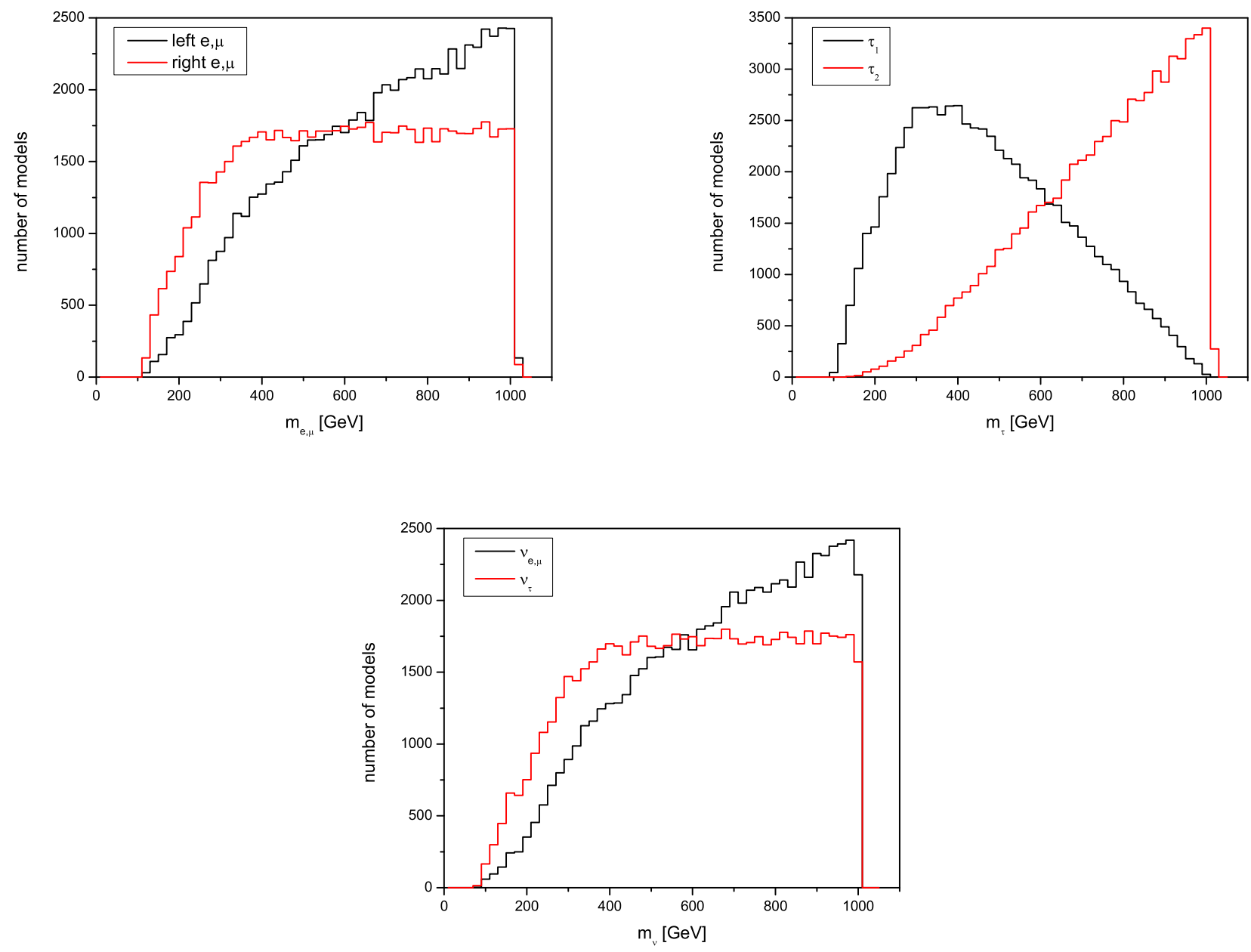

Figure 1: Distribution of slepton masses for the set of flat prior models satisfying all of our constraints: selectrons/smuons(top left), staus(top right) and sneutrinos(bottom).

Figure 3 displays the mass distributions for both neutralinos and charginos. While the LSP tends to be quite light, we see it is also common for both $\chi_{1}^{ \pm}$and $\chi_{2}^{0}$ to also to quite light with a mass almost always below $\sim 250-300 \mathrm{GeV}$. As we will see below, the almost pure Higgsino LSP scenario is reasonably common in our model set and the rough degeneracy of these three 
states then follows nearly automatically. The almost pure Wino LSP scenario is also quite common amongst our models and it also predicts a near LSP- $\chi_{1}^{ \pm}$degeneracy. Both of these scenarios can lead to long-lived charged particle signatures at the LHC. The mass distribution for gluinos, and its correlation with the LSP mass, is given in Fig. 4. Here, again, we see that many models allow for a rather light gluino, something we would not have expected based on mSUGRA due to the results of the D0 jet plus missing energy search. However, most of these light gluinos escape the D0 search due to, e.g., the smaller mass splitting with the LSP as discussed above in the case of squarks; this leads to softer jets that avoid the D0 search requirements[58].

In Fig. 5 we see that the $A, H$ and $H^{ \pm}$masses tend to track each other even for rather low mass values. A few per cent of our model set have heavy Higgs bosons which are reasonably light and are thus in the non-decoupling scenario. We note that there is a small depletion in the heavy Higgs counts when $m_{H, A, H^{+}} \simeq 2 m_{h}$. This is due to a small problem with the calculation of the $H \rightarrow 2 h$ decay width near threshold[62] in the version of SUSY-HIT that we employ. In order to avoid this problem, we have removed a small region of the phase space, leading to the depletion observed here in the $H, A$ and $H^{+}$distributions. Figure 6 shows the distribution for the lightest Higgs mass (recall there is a $3 \mathrm{GeV}$ uncertainty in the SuSpect calculation). Here we see that $(i)$ it is difficult to generate masses much above $125 \mathrm{GeV}$ (this is most likely related to our chosen parameter range that stop masses be below $\sim 1 \mathrm{TeV}$ ), and $(i i)$ a small set of models predict light Higgs bosons with masses as low as $85-90 \mathrm{GeV}$. Clearly in such models the Higgs couplings are either quite different than in the SM with, e.g., reduced ZZh couplings with a lighter $m_{A}$ (such as in the non-decoupling region), and/or the light Higgs decays into LSP pairs with a large branching fraction (while simultaneously avoiding the constraint on $Z$ decay into invisible particles). In fact, we find that both situations do occur in our set of models. We note in passing that we encounter a second SUSH-HIT issue in the case of very light Higgs in the mass range between 80 and 90 $\mathrm{GeV}$. Here, an anomalously large partial width for the $\gamma \gamma$ mode is returned by the code yielding an invalid result. These few cases appear in both the flat and log prior samples and are dropped 
from our final model sample.
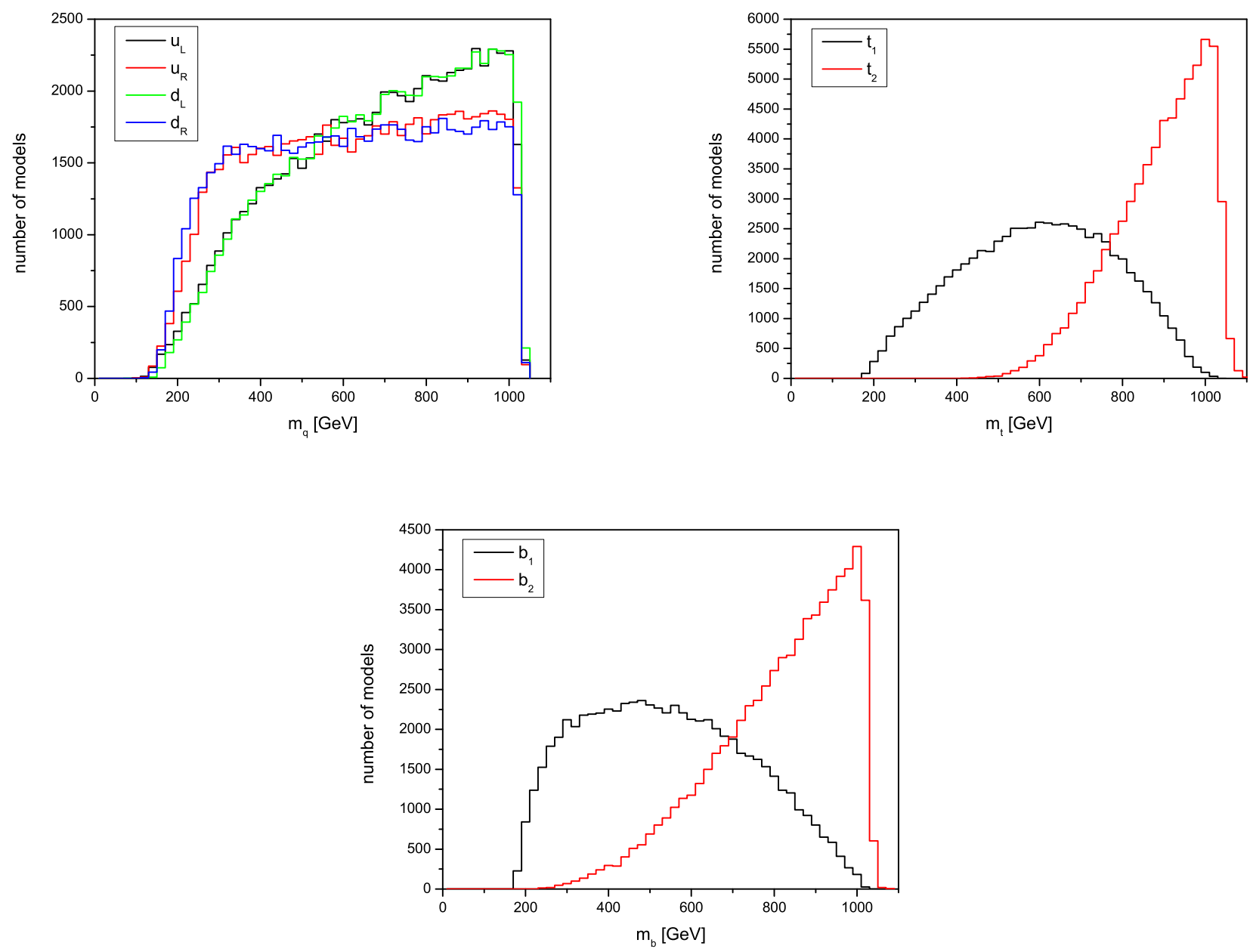

Figure 2: Distribution of squark masses for the set of flat prior models satisfying all of our constraints: first/second generation squarks are in the upper left panel, stops in the upper right panel and sbottoms are in the lower panel.

The properties of the nLSP are examined in Fig. 7, which shows the various possible identities of the nLSP in our surviving flat prior model set, as well as the mass splitting between the LSP and the nLSP as a function of the LSP mass. These two properties essentially determine the collider phenomenology of a model. From this figure, we see that the lightest chargino plays the role of the nLSP much of the time but many of the other sparticles can also be the nLSP at the level of a few percent. The second most common nLSP is found to be $\chi_{2}^{0}$ occurring in $\sim 6-7 \%$ of 

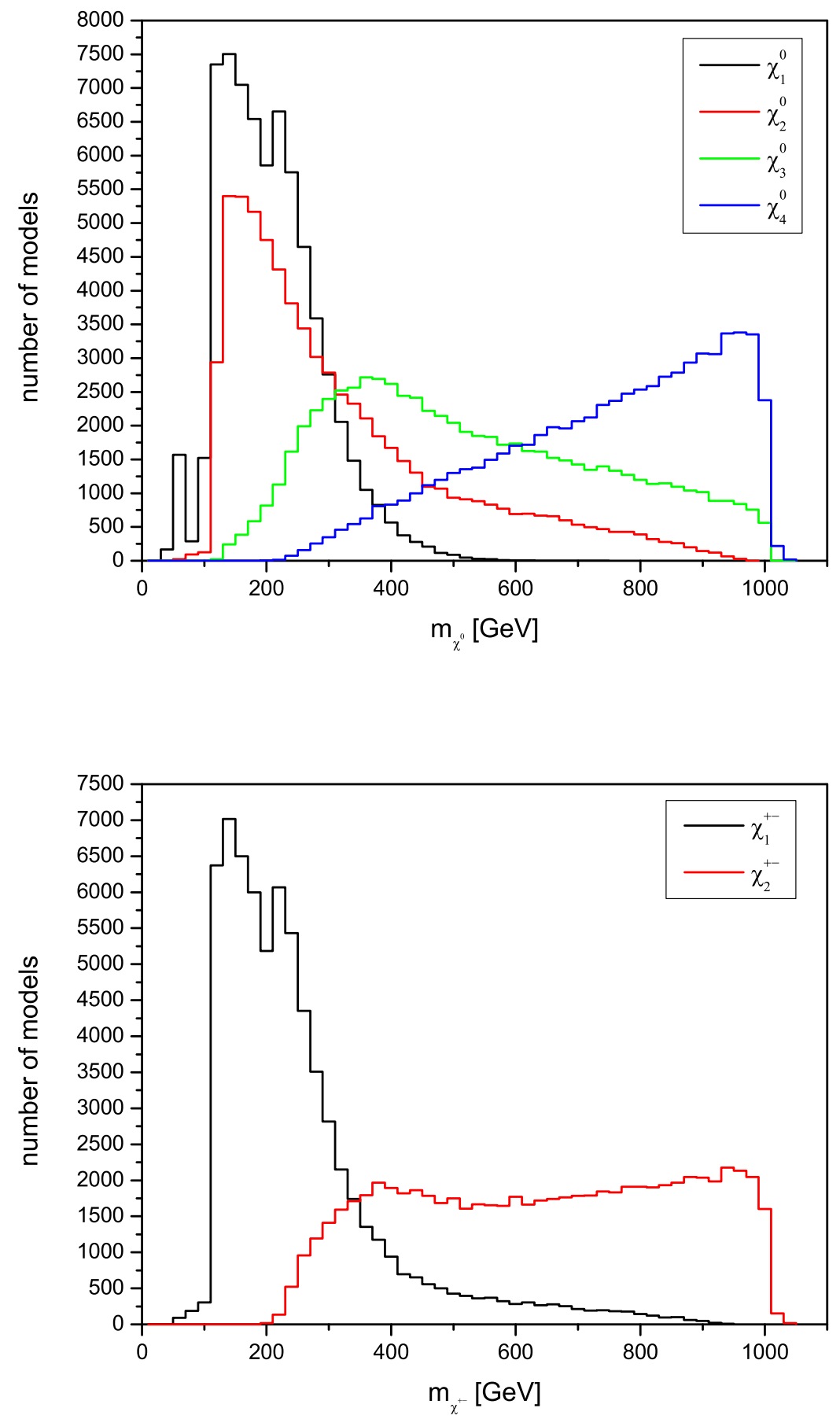

Figure 3: Distribution of neutralino and chargino masses for the set of flat prior models satisfying all of our constraints. 

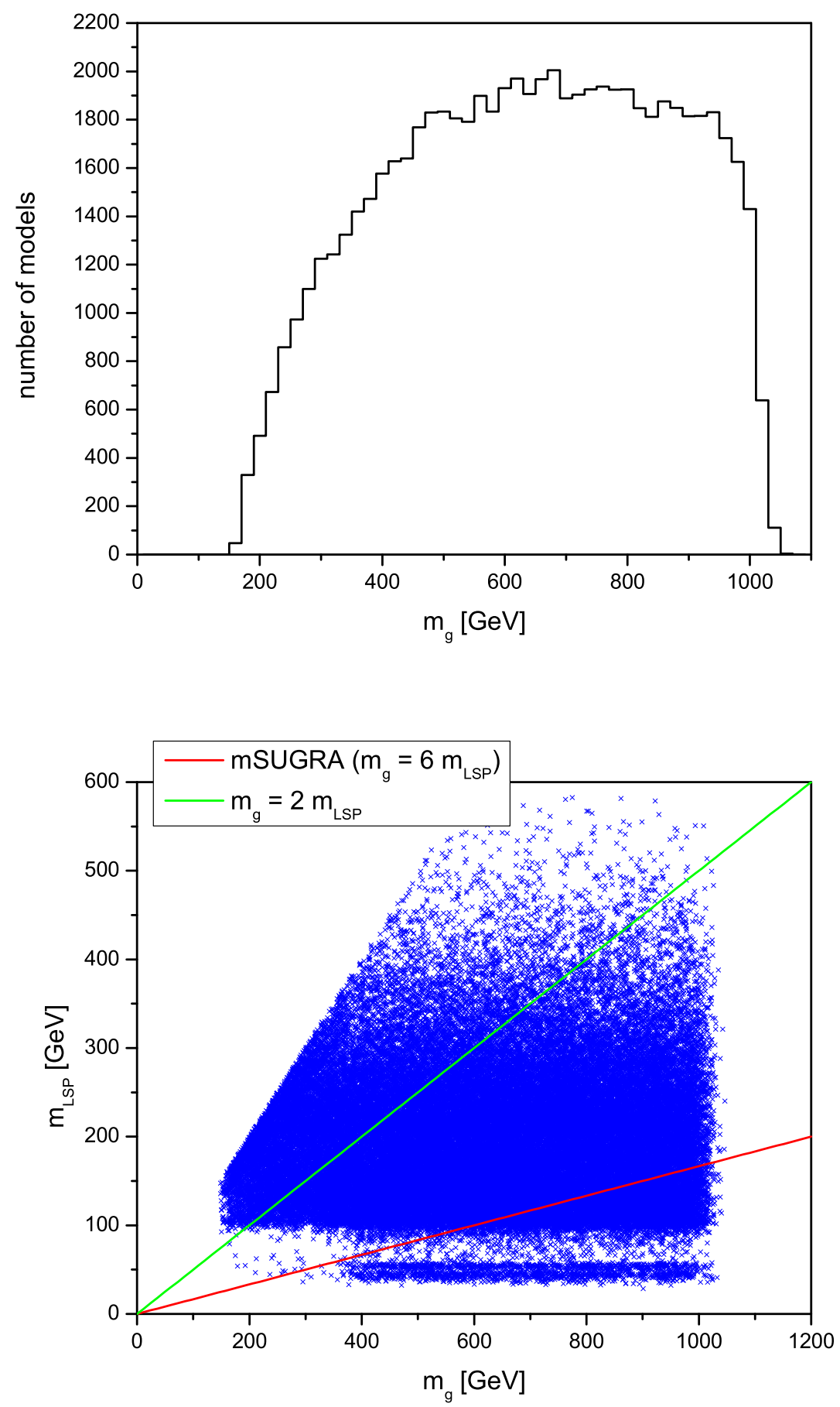

Figure 4: Distribution of gluino masses and a comparison of the gluino and LSP masses for the set of flat prior models satisfying all of our constraints. 

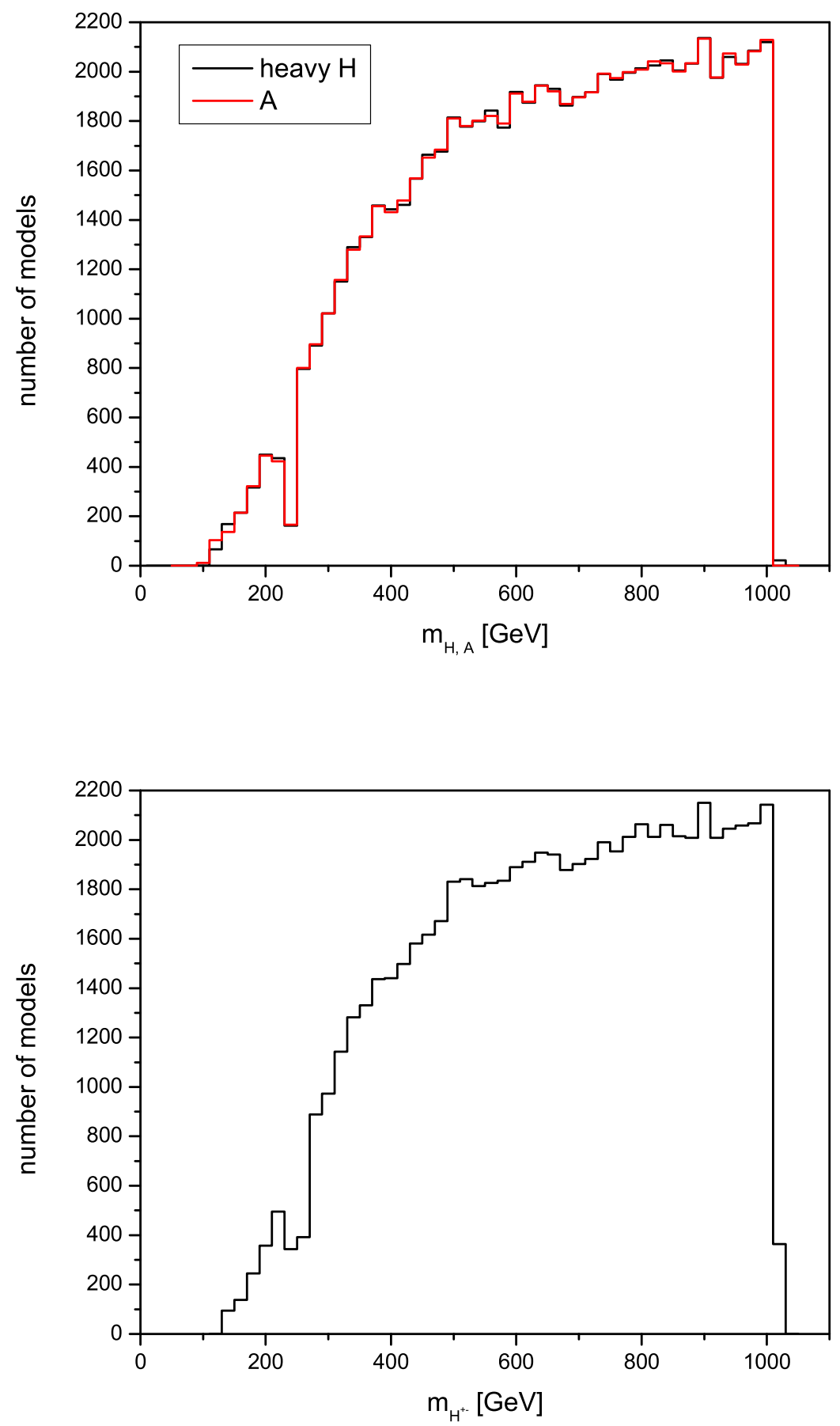

Figure 5: Distribution of heavy and charged Higgs masses for the set of flat prior models satisfying all of our constraints. 


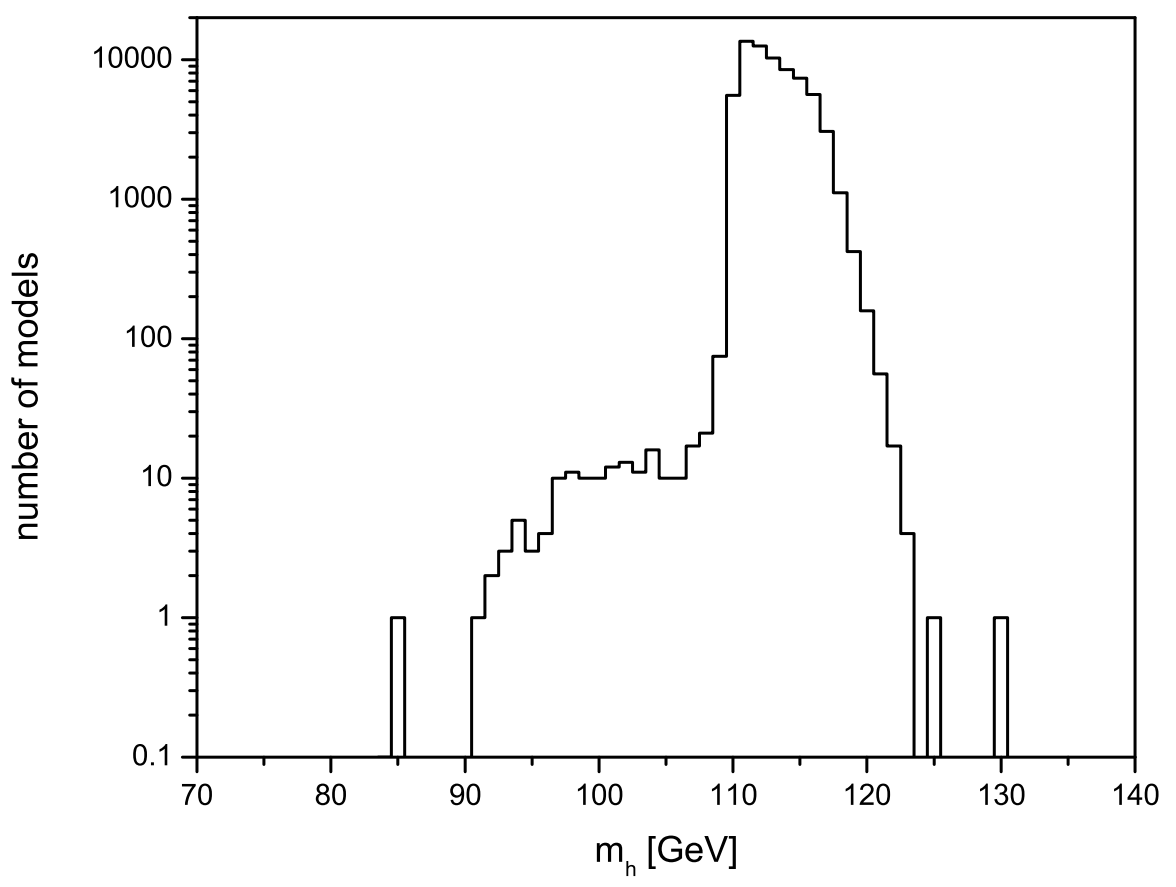

Figure 6: Distribution of the light Higgs mass on a log scale for the set of flat prior models satisfying all of our constraints. 
the cases; these are mainly pure Higgsino LSP scenarios. Other identities of the nLSP may lead to unusual signatures at the LHC. In the lower panel of this figure we see not only the nLSP identity but also the nLSP-LSP mass splitting, $\Delta m$, as a function of the LSP mass itself. Here we see that there is a strong concentration of models with small values of $\Delta m$ in our sample; in hindsight this is not too surprising since, as we'll see below, the LSP in our model set is commonly either pure Wino or Higgsino or a small admixture of these two cases. The bulk of the mostly empty square region which appears on the lower left-hand side of the figure has been removed by the stable chargino search at the Tevatron; only non-chargino nLSPs remain in this region**. Again, we emphasize that the LSP in the models with small values of $\Delta m$ tend much of the time to be close to a Wino or Higgsino (or combination thereof) electroweak eigenstate as we will see below. We note that a stable heavy charged particle search at the LHC should exclude or discover the models with heavier Chargino nLSPs and small values of $\Delta m$. There are many interesting features worth noting in the lower panel of this figure. For example, we see that there are a cluster of models with light charginos, i.e., masses less than $100 \mathrm{GeV}$, but with small ( $\lesssim 1 \mathrm{GeV}$ ) values of $\Delta m$. Such particles, though sufficiently short-lived, would only leave soft tracks in a LEP detector and would likely to have been missed. We also see a set of models with a very small $\Delta m$ where $\chi_{2}^{0}$ is the nLSP but with masses such that $Z \rightarrow \chi_{2}^{0} \chi_{1}^{0}$ is kinematically forbidden. Again, such final states would be nearly impossible to observe and would have been missed at LEP. This figure also reveals cases with light squark nLSPs and small values of $\Delta m$. Furthermore, we observe that models in this figure with the lightest LSPs and also have larger values of $\Delta m \sim 10-300 \mathrm{GeV}$, can have almost any SUSY particle as the nLSP. The small gap in the region $1 \lesssim \Delta m \lesssim 30$ for LSP masses $\lesssim 100$ GeV may be easily explained. One possibility is that in the many models where we encounter an almost pure Wino or Higgsino LSP with a small mass difference with a chargino nLSP, the mass of such a chargino must be in excess of $\sim 100 \mathrm{GeV}$ due to LEP constraints. Bino LSPs, on the otherhand, are not subject to such constraints and can be much lighter though are found to be

\footnotetext{
** Note that sleptons with masses in this range have cross sections which are too small to beexcluded by the Tevatron search.
} 
somewhat less common in our sample..

Based on these results there are many interesting possible scenarios that can arise at the LHC. Here, we give two examples involving small mass splittings between the nLSP and LSP. In the first case we consider, we find that there are a class of models with a squark nLSP with very light masses $\sim 80-120 \mathrm{GeV}$ and mass splittings from the LSP of only a few GeV or less. In the low end of this squark mass range, such events would have appeared as a two-photon background at LEP, whereas for the full mass range the relative softness of the jets in the final state would imply that the squarks would have been missed by the D0 multijet search. Such sparticles would be notoriously difficult to detect at the LHC; it is perhaps possible that using event samples with additional gluon ISR would be useful[58] but would require further study. A second interesting scenario is the case where $\tilde{\chi}_{2}^{0}$ is the nLSP having a small mass splitting with the LSP. The properties of $\tilde{\chi}_{2}^{0}$ neutralinos are usually determined via the cascade decays of heavier colored sparticles produced with large cross sections or when they are produced in association with the lightest chargino via the trilepton mode discussed above. The most common case where $\tilde{\chi}_{2}^{0}$ is the nLSP in our model sample is for both it and the LSP to be dominantly Higgsino-like, further implying that the lightest chargino would also be nearby in mass. In this situation, due to the Higgsino-like nature, we would not expect significant trilepton production and the appearance of $\chi_{2}^{0}$ in cascade decays would be most unusual. If $\tilde{\chi}_{2}^{0}$ is produced in a cascade decay (by, say, stop pair production followed by the decay $\left.\tilde{t}_{1} \rightarrow \tilde{\chi}_{2}^{0}\right)$, the $\tilde{\chi}_{2}^{0}$ decay to the LSP would be controlled by the size of the allowed phase space which may be well below $1 \mathrm{GeV}$ in some cases leading to a possible secondary vertex. For some mass splittings, decays through a virtual $Z$ or $h$ may dominate leading to an additional pair of (b)jets or leptons. In other cases, decays into a photon would be dominant leading to a final state with additional non-pointing photons.

Since the nLSP can be almost any SUSY particle, and the corresponding mass splittings can be small in all cases, essentially all of the conventional SUSY long-lived particle scenarios can be captured in our model set. For example, long-lived stops or staus (as in GMSB[5]), gluinos (as in 
Split SUSY[59]) as well as charginos (as in AMSB[6]) all occur in our sample as do other possibilities including long-lived selectrons, sneutrinos and sbottoms. Of course, long-lived $\tilde{\chi}_{1}^{0}$ neutralinos can occur in GMSB, where they are the nLSP, whereas here long-lived neutralinos can only be the $\tilde{\chi}_{2}^{0}$.

As an aside, it is interesting to note that the set of mass spectra obtained above for the various sparticles, as well as the large number of models with small nLSP-LSP mass splittings, are qualitatively similar to the corresponding ones in a set of models given in Ref. [60]. These models were found to be difficult to distinguish at the LHC and were subsequently further analyzed in detail in the ILC context[61]. As we will see below, this similarity will remain valid in the case of our models generated via log priors as well. This present analysis thus demonstrates that there is nothing special (or wrong) about that particular[60] model set.

Figure 8 shows the detailed Wino/Higgsino/Bino content of the LSP in our set of models. Here we see that, as advertised, the LSPs in our sample tend to be either a rather pure electroweak eigenstate or a mixture of only two of these states. LSPs with large weak eigenstate admixtures, that would populate the central regions of these figures, are seen to be relatively rare in our model sample. In addition, Wino-like and Higgsino-like LSPs are seen to be somewhat dominant over the Bino-like case familiar from mSUGRA.

Let us now turn to the pMSSM postdictions, i.e., the distribution of values from our model set for the experimental quantities used as constraints in obtaining that model set. Figure 9 displays these postdictions for several of the experimental observables, as well as for the values of $\tan \beta$, from our surviving model sample. Note that this particular distribution peaks at values near $\tan \beta \simeq 12$ and favors smaller values of this quantity. As expected, the distribution for $\delta(g-2)_{\mu}$ is approximately bimodal, depending upon the sign of the Higgsino mixing parameter $\mu$. Here, we see that most models do not predict a large enough shift in this observable to fully 'explain' the central value of the observed discrepancy with the SM. We also see that while there is a slight preference for somewhat larger values of $B(b \rightarrow s \gamma)$ the predicted values for $B\left(B_{s} \rightarrow \mu^{+} \mu^{-}\right)$lie significantly below the current Tevatron bound and hover around the SM prediction for this decay. 

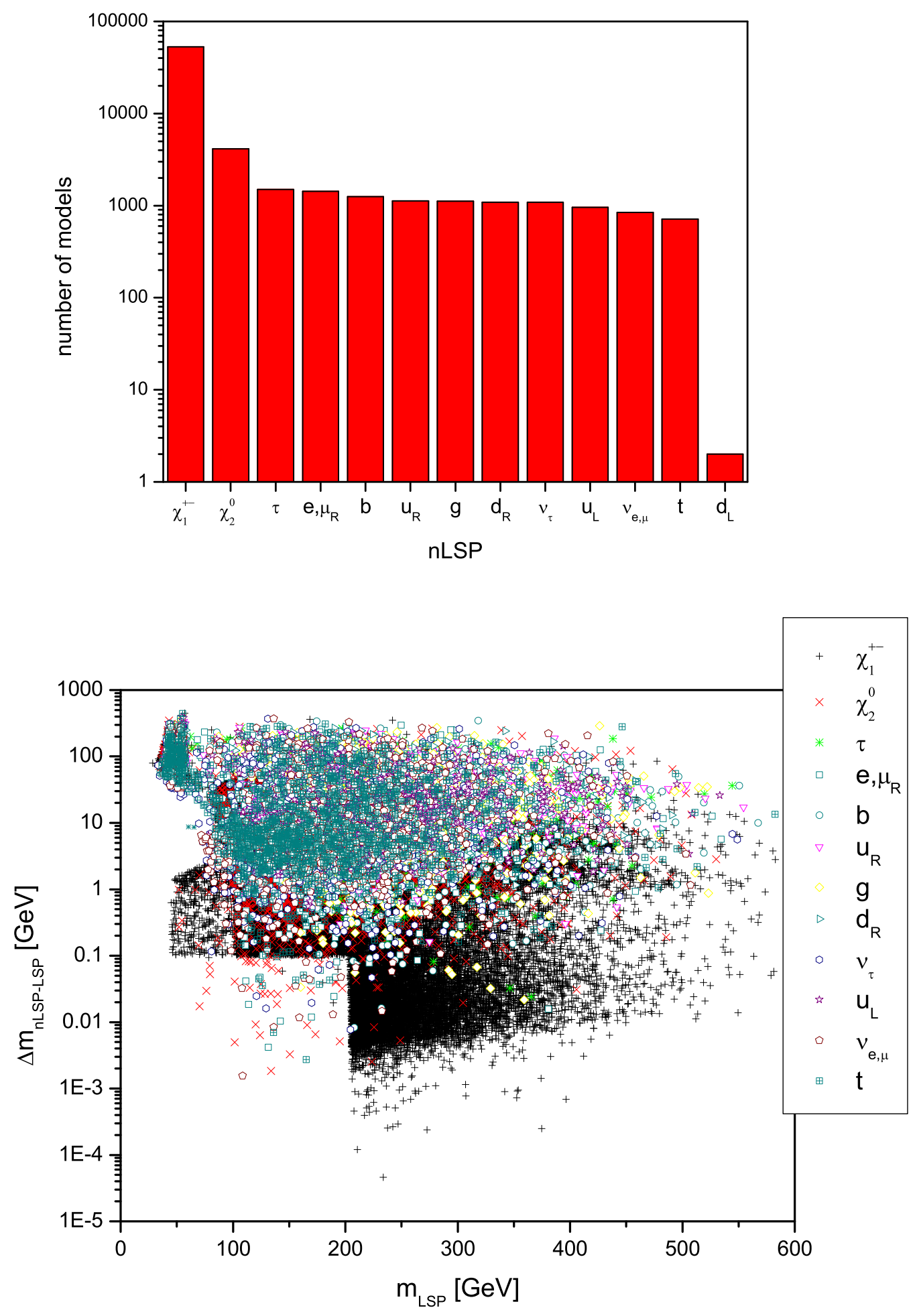

Figure 7: (Top) Identity of the nLSP. (Bottom) nLSP-LSP mass splitting as a function of the LSP mass, with the identity of the nLSP as labeled. Both assume flat priors. 

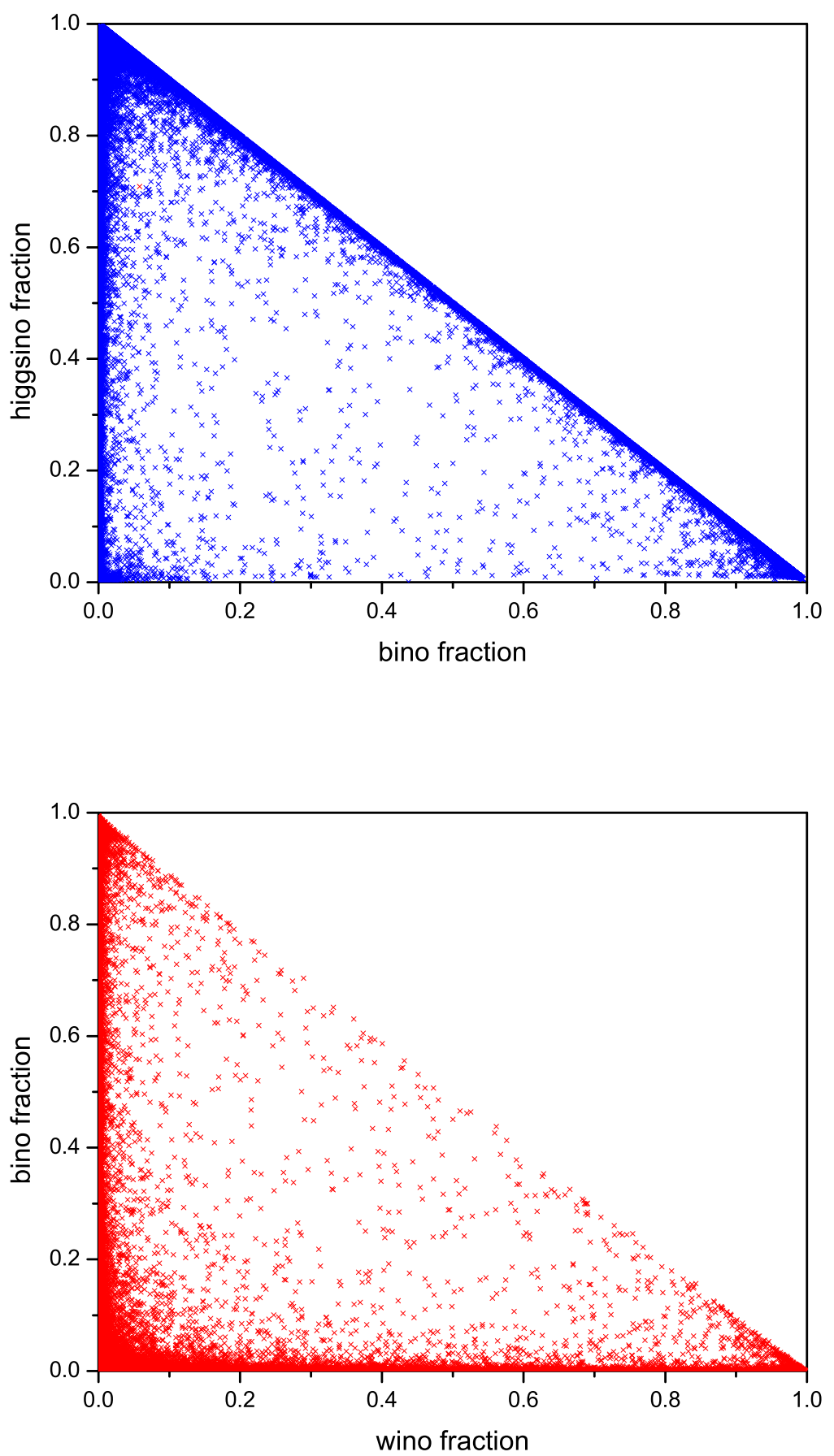

Figure 8: Wino/Higgsino/Bino content of the LSP in the case of flat priors. 
Generally, $B(B \rightarrow \tau \nu)$ is not found to be a great discriminator amongst our model set. Note that the distribution for the predicted value of the relic density $\Omega h^{2}$ is peaked at rather small values implying that in most of our models the dark matter must be dominantly non-LSP in origin. However we also see that there is a long tail leading to a significant number of models with $\Omega h^{2}$ values close to the WMAP upper limit.

We show a number of relevant predictions for dark matter in our flat prior model sample Fig. 10. In the top panels we see the prediction for $\Omega h^{2}$ as a function of both the LSP mass as well as the LSP-nLSP mass splitting, $\Delta m$. Note that the predicted range for $\Omega h^{2}$ is quite large but is found to peak near $\sim 0.004$, which is about $1 / 30$ of the observed value obtained by WMAP as discussed above. These models then would require that there be a substantial amount of dark matter from other sources such as axions. The range of predictions presented here for the relic density is found to be much larger than those obtained by other analyses[22] that were restricted to specific SUSY breaking scenarios. We also show the scaled spin-independent as well as spindependent LSP-proton scattering cross sections relevant for direct detection experiments. Note that again the predicted ranges that we find for these scattering cross sections are far larger than those based on mSUGRA, as obtained by, e.g., Ref.[63]. In fact, we find that the range of values we obtain from our pMSSM model set essentially covers the entire theoretical anticipated region found in this reference for all different types of theories beyond the SM. Thus, this observable can not be used as a model discriminator, e.g., to distinguish between SUSY versus Universal Extra Dimenions or Little Higgs thermal relics. Clearly these results show that the range of expectations from the pMSSM is far larger than those predicted within any specific SUSY breaking scenario.

The amount of fine-tuning in our model sample is described in Fig. 11. Here we see that there is a clear set of pMSSM models with a rather reasonable degree of fine-tuning, unlike the current situation within mSUGRA[22]. Following the analysis presented in Ref. [64], we define the 

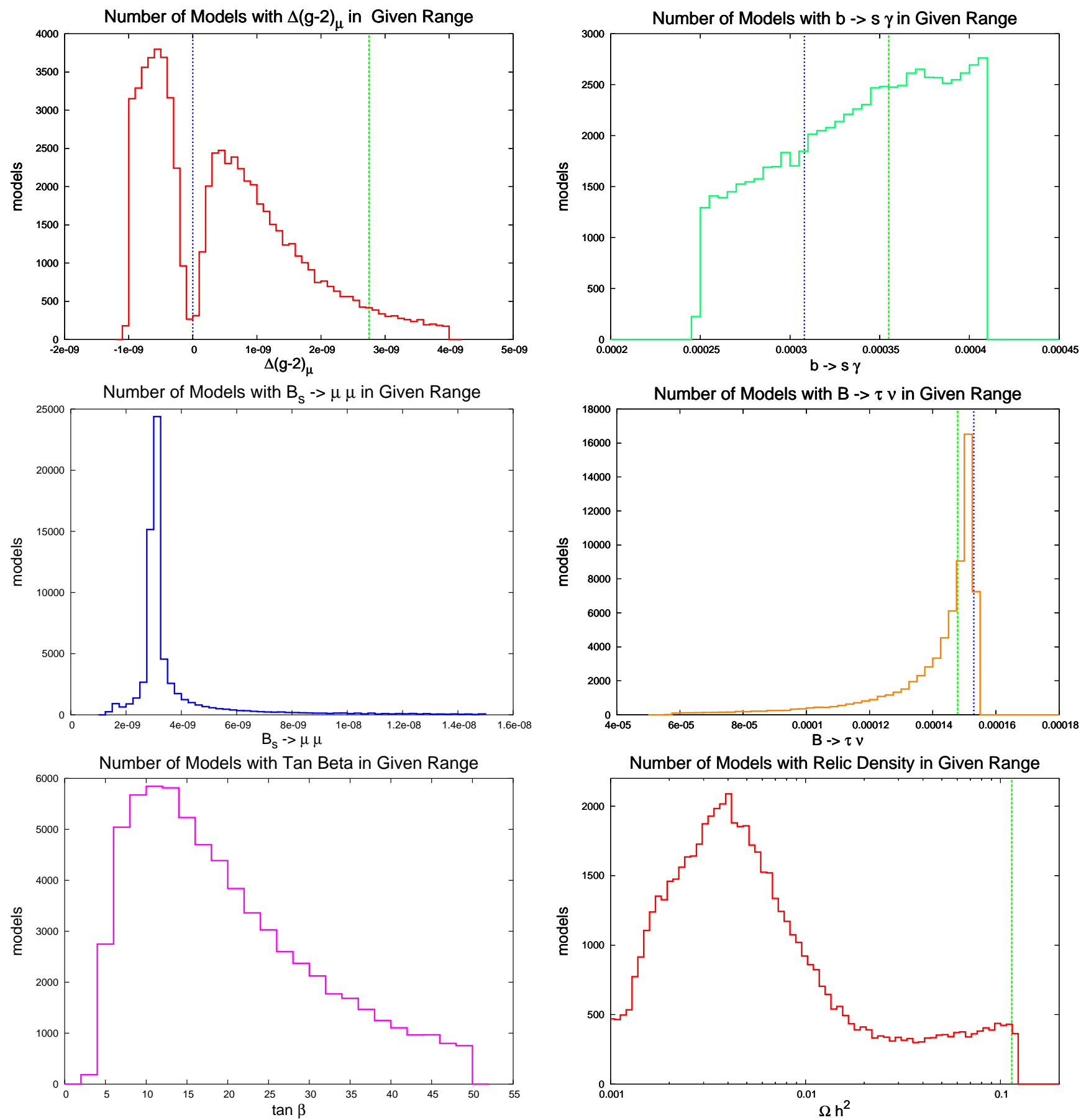

Figure 9: Distributions of predictions for several observables as well as $\tan \beta$ for our model sample subject to the constraints discussed in the text. The blue and green dashed lines show the SM predictions as well as the current central values obtained by experiment, respectively. 

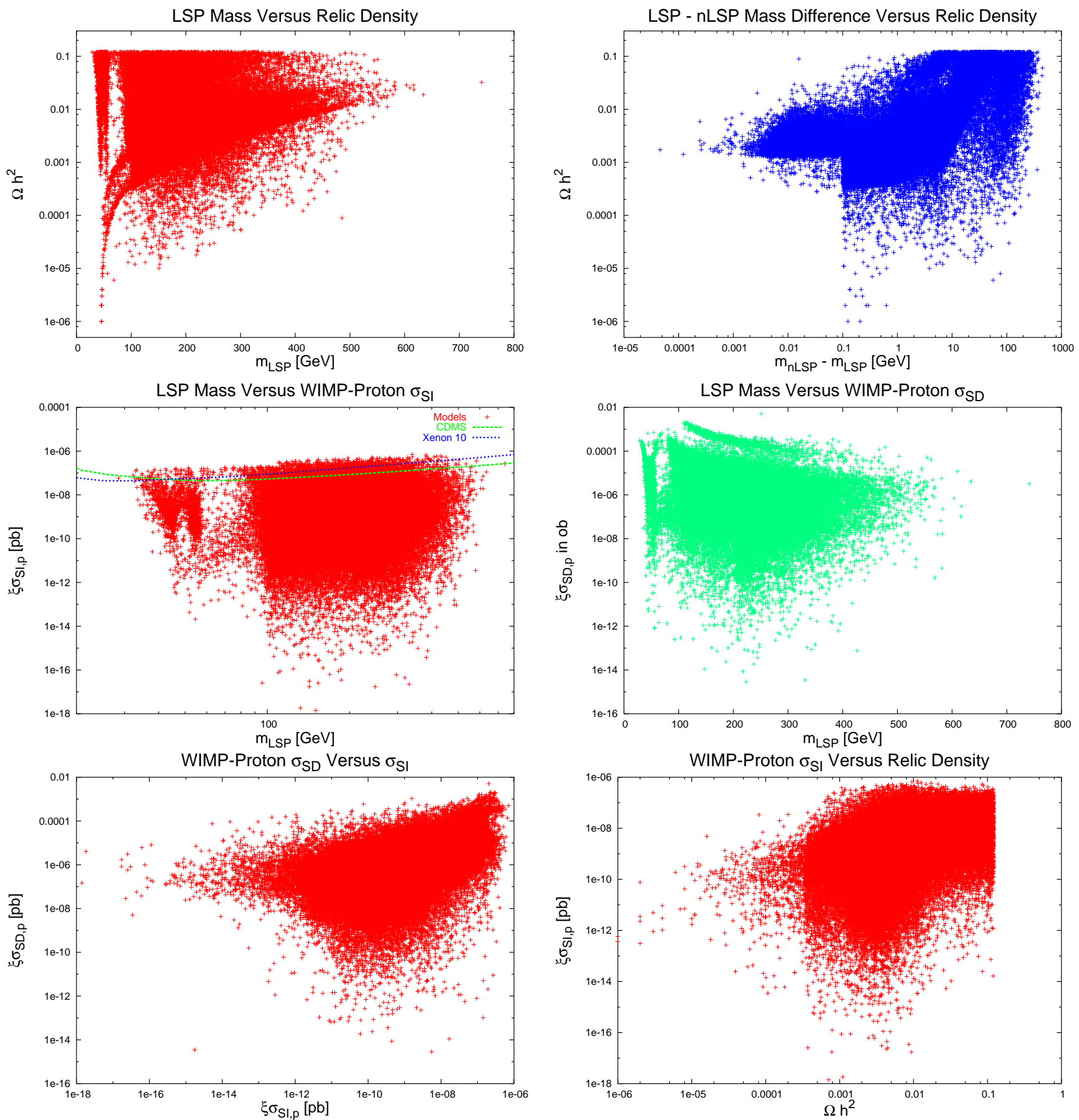

Figure 10: Distributions of relevant dark matter predictions from our model sample subject to the constraints discussed in the text. All cross sections are in pb. 
quantities

$$
A(\xi)=\left|\frac{\partial \log m_{Z}^{2}}{\partial \log \xi}\right|
$$

where $\xi$ are the set of relevant Higgs Lagrangian parameters within the pMSSM. Then, for example

$$
\begin{aligned}
A(\mu) & =\frac{4 \mu^{2}}{m_{Z}^{2}}\left(1+\frac{m_{A}^{2}+m_{Z}^{2}}{m_{A}^{2}} \tan ^{2} 2 \beta\right), \\
A(b) & =\left(1+\frac{m_{A}^{2}}{m_{Z}^{2}}\right) \tan ^{2} 2 \beta, \\
A\left(m_{u}^{2}\right) & =\left|\frac{1}{2} \cos 2 \beta+\frac{m_{A}^{2}}{m_{Z}^{2}} \cos ^{2} \beta-\frac{\mu^{2}}{m_{Z}^{2}}\right| \times\left(1-\frac{1}{\cos 2 \beta}+\frac{m_{A}^{2}+m_{Z}^{2}}{m_{A}^{2}} \tan ^{2} 2 \beta\right), \\
A\left(m_{d}^{2}\right) & =\left|-\frac{1}{2} \cos 2 \beta+\frac{m_{A}^{2}}{m_{Z}^{2}} \sin ^{2} \beta-\frac{\mu^{2}}{m_{Z}^{2}}\right| \times\left|1+\frac{1}{\cos 2 \beta}+\frac{m_{A}^{2}+m_{Z}^{2}}{m_{A}^{2}} \tan ^{2} 2 \beta\right|,
\end{aligned}
$$

where it is has been assumed that $\tan \beta>1$. Here, $m_{u, d}^{2}$ are the effective mass parameters for the two Higgs doublets generating masses for the $u, d$-type quarks, respectively, and $b$ is the parameter in the Higgs potential that can be rewritten in terms of $\tan \beta$ and $m_{A}$. The overall fine-tuning $\Delta$ is defined by adding these four quantities in quadrature. Values of $\Delta$ far above unity then indicate large amounts of fine-tuning. In this figure we see that a reasonable set of our surviving models can have very low values of finetuning. This figure also shows that the overall amount of fine-tuning

indeed peaks at rather low values in our set of models, far smaller than that obtained in the case of mSUGRA. Note that the fine-tuning percentage given in the bottom panel of this figure is simply the value of $1 / \Delta$ expressed as a per-cent.

\subsection{Log Priors}

In the case where we employed log priors, a far smaller number (and fraction) of models survive the confrontation with the experimental constraints. In this case, we randomly sampled $2 \cdot 10^{6}$ models and found that only $\sim 3000$ models remained after imposing the theoretical and experimental 

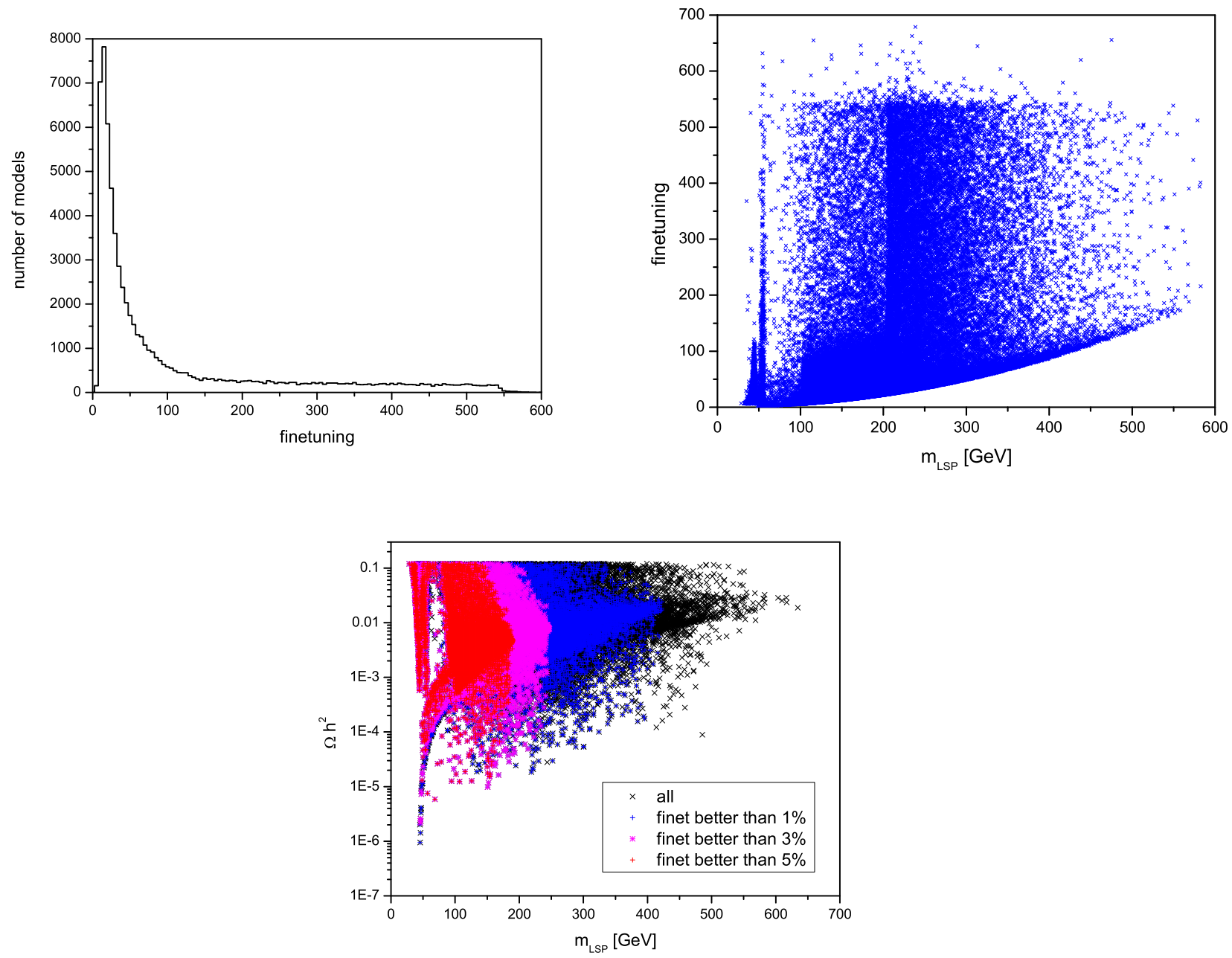

Figure 11: The distribution of fine-tuning $(=\Delta)$ in our set of models and as a function of the LSP mass. Also shown is the correlation of the amount of fine-tuning with the predicted dark matter density. 
constraints. The main reason for this is rather straightforward to understand. In the log prior scan, although the range of the SUSY masses has been expanded, most of the generated parameter space points correspond to models with very light SUSY particles which are more highly constrained by existing experimental data and, consequently, far fewer of the models survive. Recall that we employed a smaller model sample in this case as our primary goal in undertaking the log prior study is to examine any differences with the flat prior analysis and consequently our presentation of the corresponding results will be somewhat brief.

We begin our comparison with the flat prior results by examining the various Higgs and sparticle spectra. Figures $12-17$ show the set of spectrum results for the set of log prior models which survive all of the constraints. These should be compared to Figs. 1- 6 above in the case of flat priors. As can be seen these spectra are qualitatively very similar to what we have seen in the case of flat priors above, except for the obvious two effects already mentioned: the ranges of the allowed mass values have been extended so that larger masses are reached and the sample size is significantly smaller. However, the broad features discussed above for the flat prior sample also hold true for the log prior sample. The main feature that we observed in both log and flat prior samples is the apparent preference for relatively light spartners with this preference being somewhat stronger in the log prior case as would be expected. While the low end of the log prior distributions are similar to those in the flat prior analysis, naturally, the log prior spectra have longish tails which stretch out to significantly larger mass values. Larger $A$-terms, for example, can lead to greater stop mixing effects which then produce larger values for the light Higgs mass; this also leads to a slight increase in the maximum value of the Higgs mass in comparison to the flat prior case, as is observed in Fig. 17.

Figures. 18 and 19 describe the nature of the nLSP and the corresponding mass splitting with the LSP for the case of log priors, as well as the associated electroweak origin of the LSP. While different in some details from the flat prior results, here we see that the properties of the LSP and the possible identities of the nLSP are essentially the same in the two cases. The slight difference 

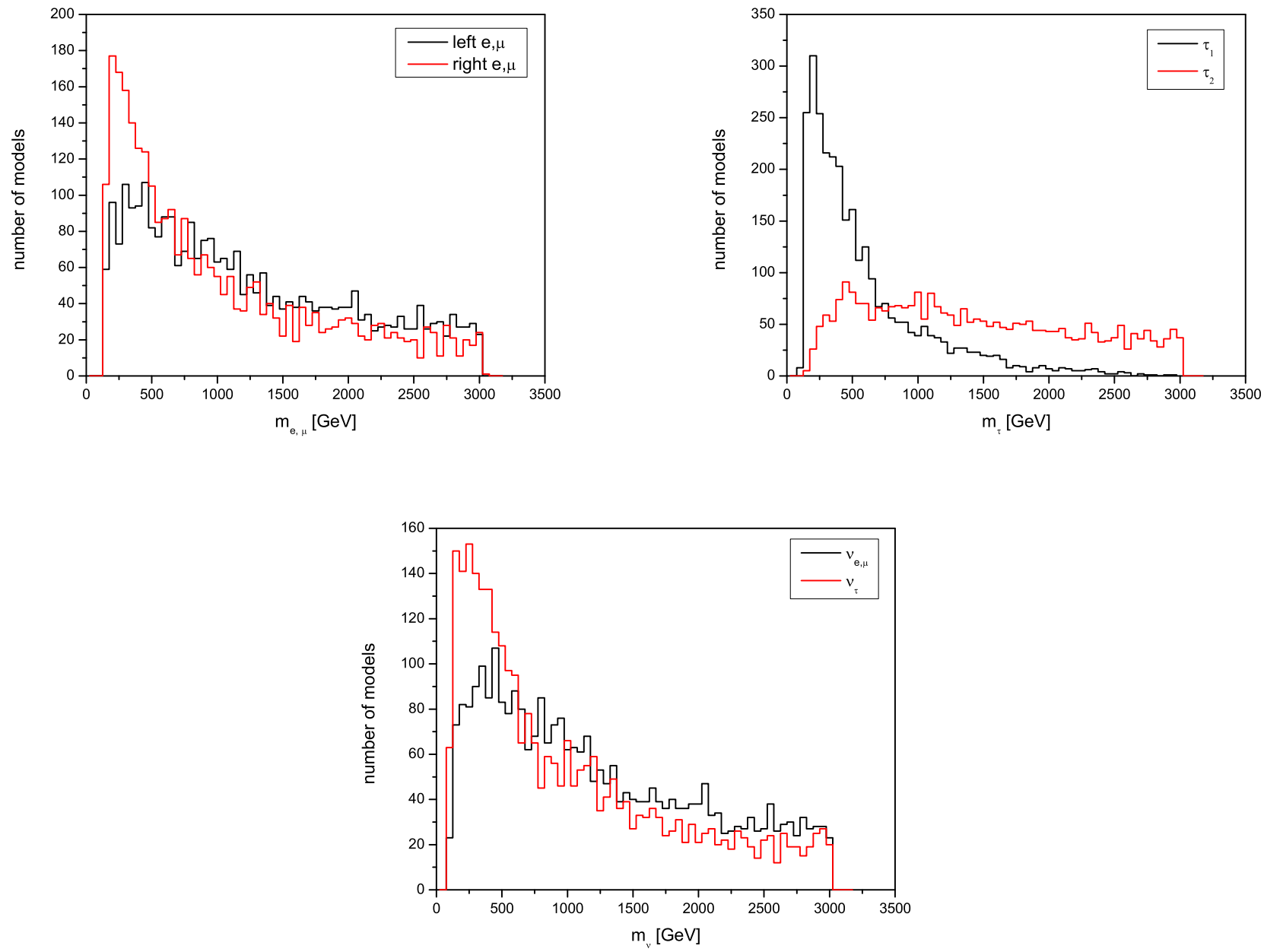

Figure 12: Distribution of slepton masses for the set of log prior models satisfying all of our constraints: selectrons/smuons(top left), staus(top right) and sneutrinos(bottom). 

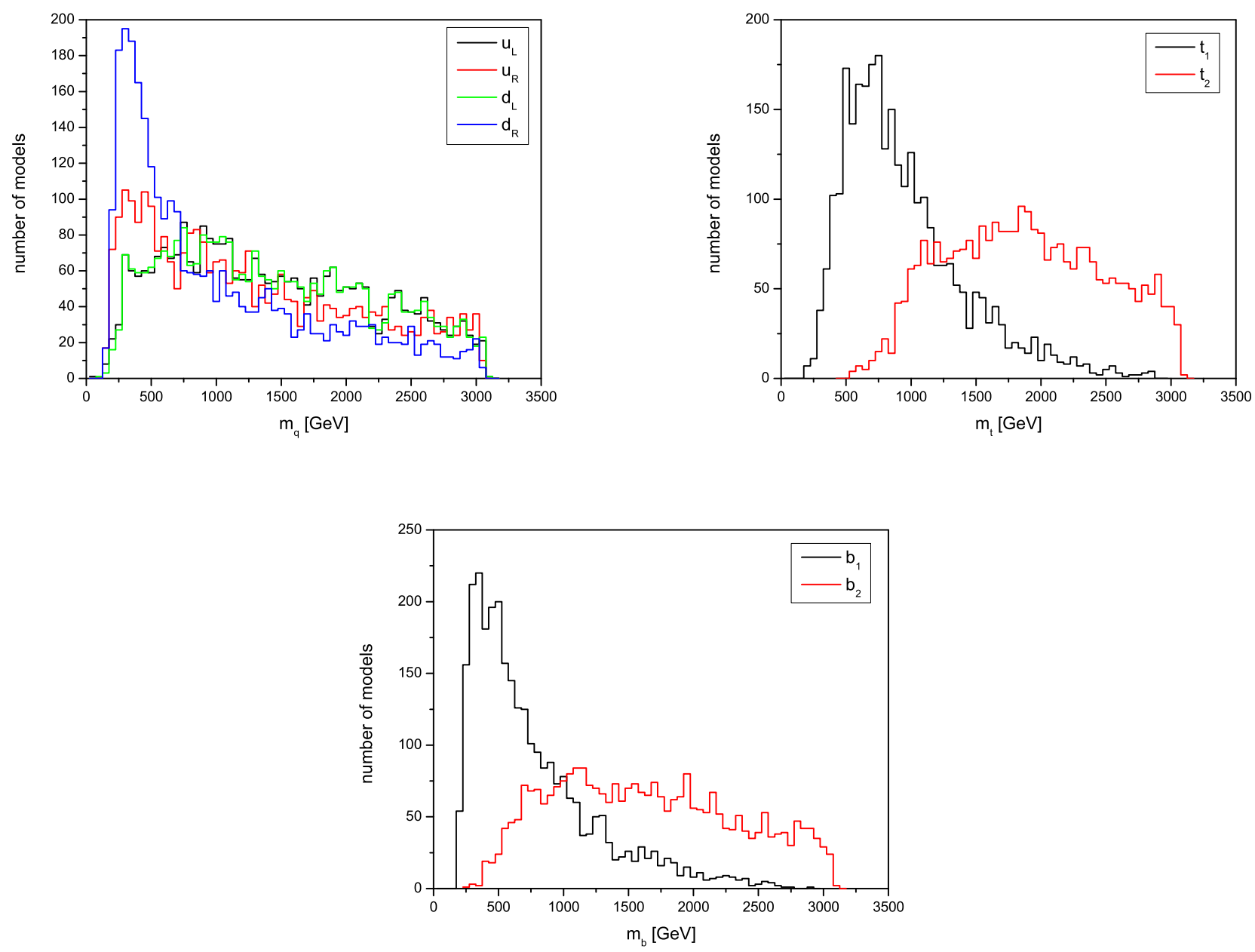

Figure 13: Distribution of squark masses for the set of log prior models satisfying all of our constraints: 'light' (first/second generation) squarks are in the upper left panel, stops in the upper right panel and sbottoms are in the lower panel. 

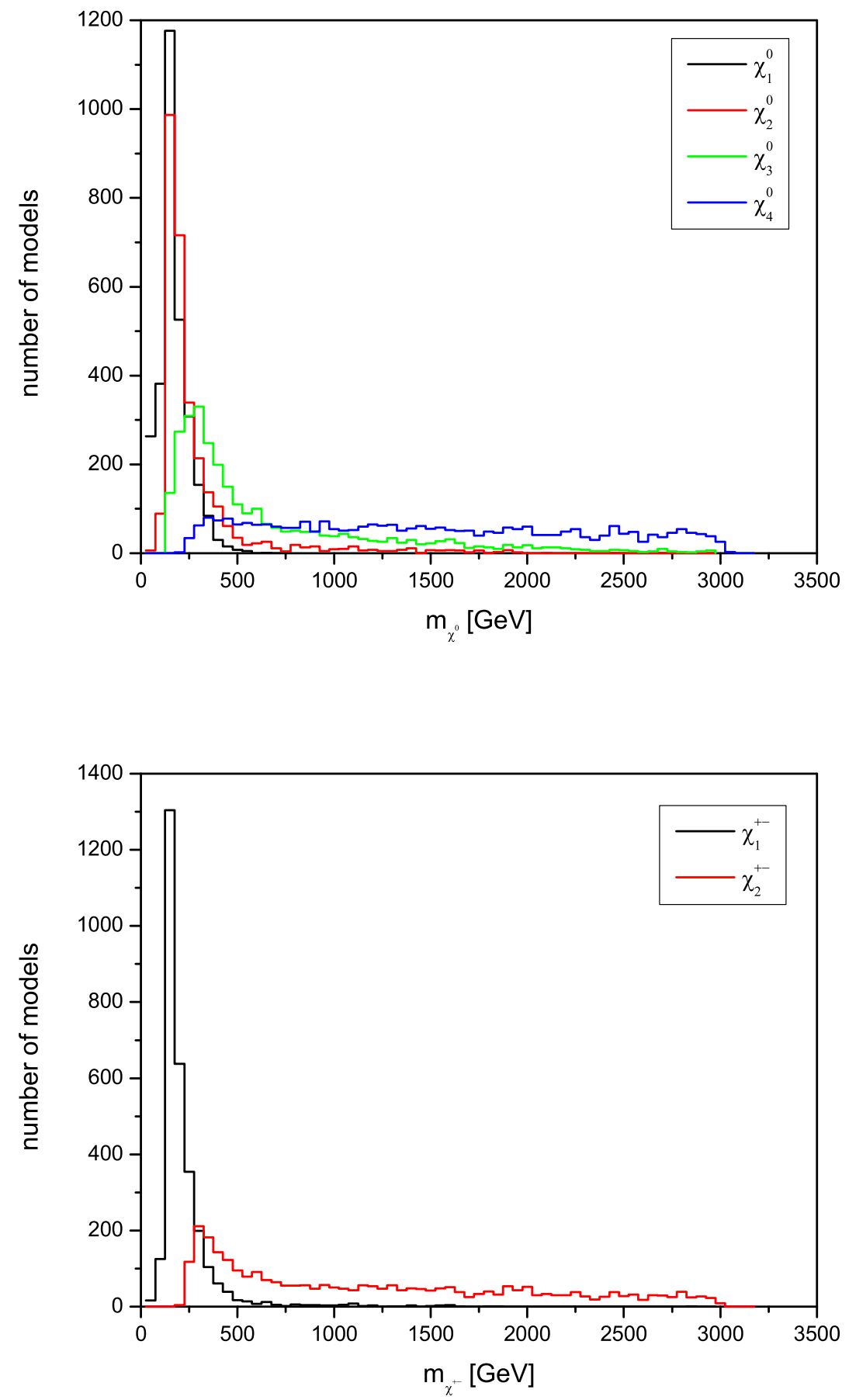

Figure 14: Distribution of neutralino and chargino masses for the set of log prior models satisfying all of our constraints. 

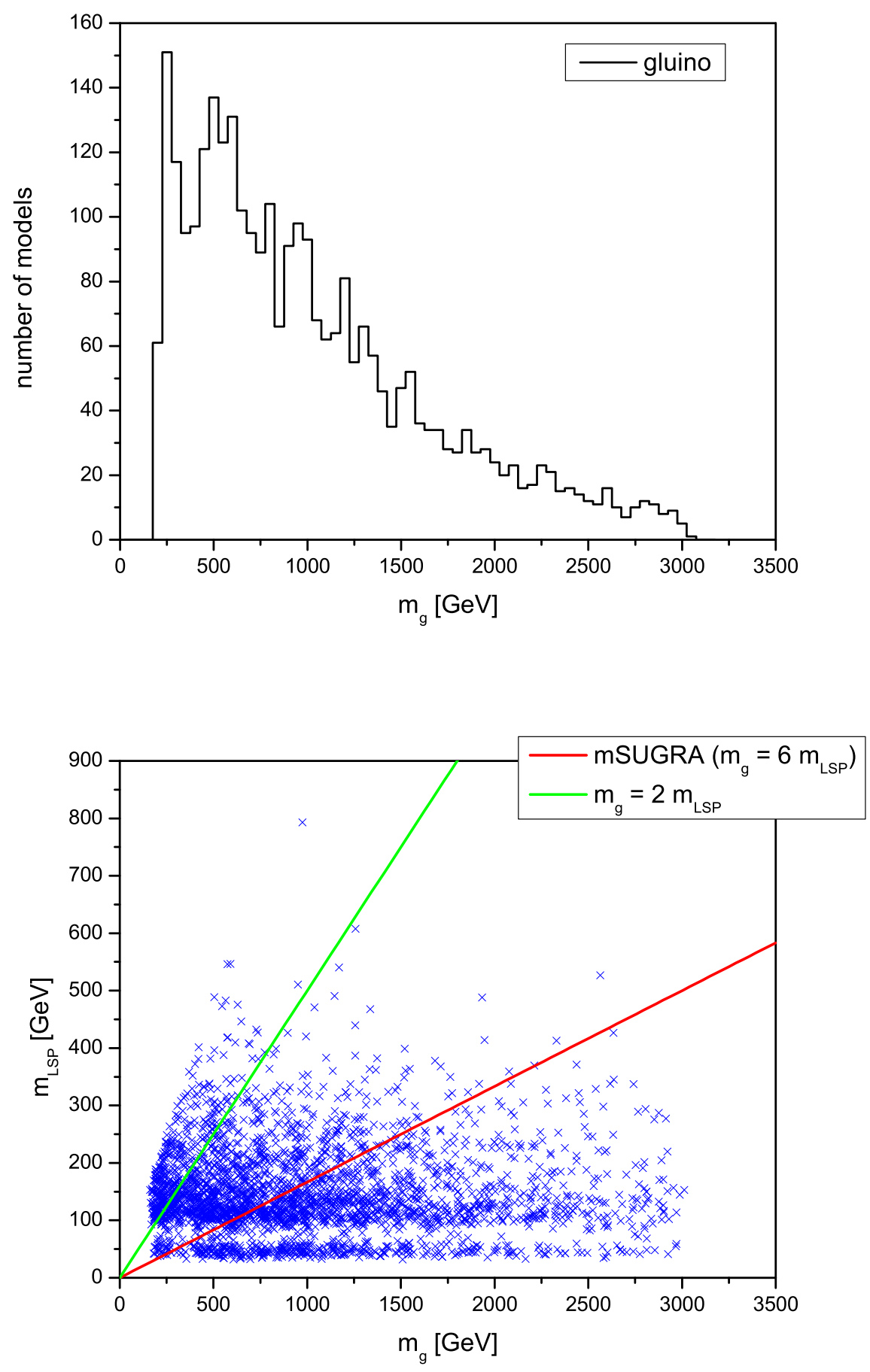

Figure 15: Distribution of gluino masses and a comparison of the gluino and LSP masses for the set of log prior models satisfying all of our constraints. 

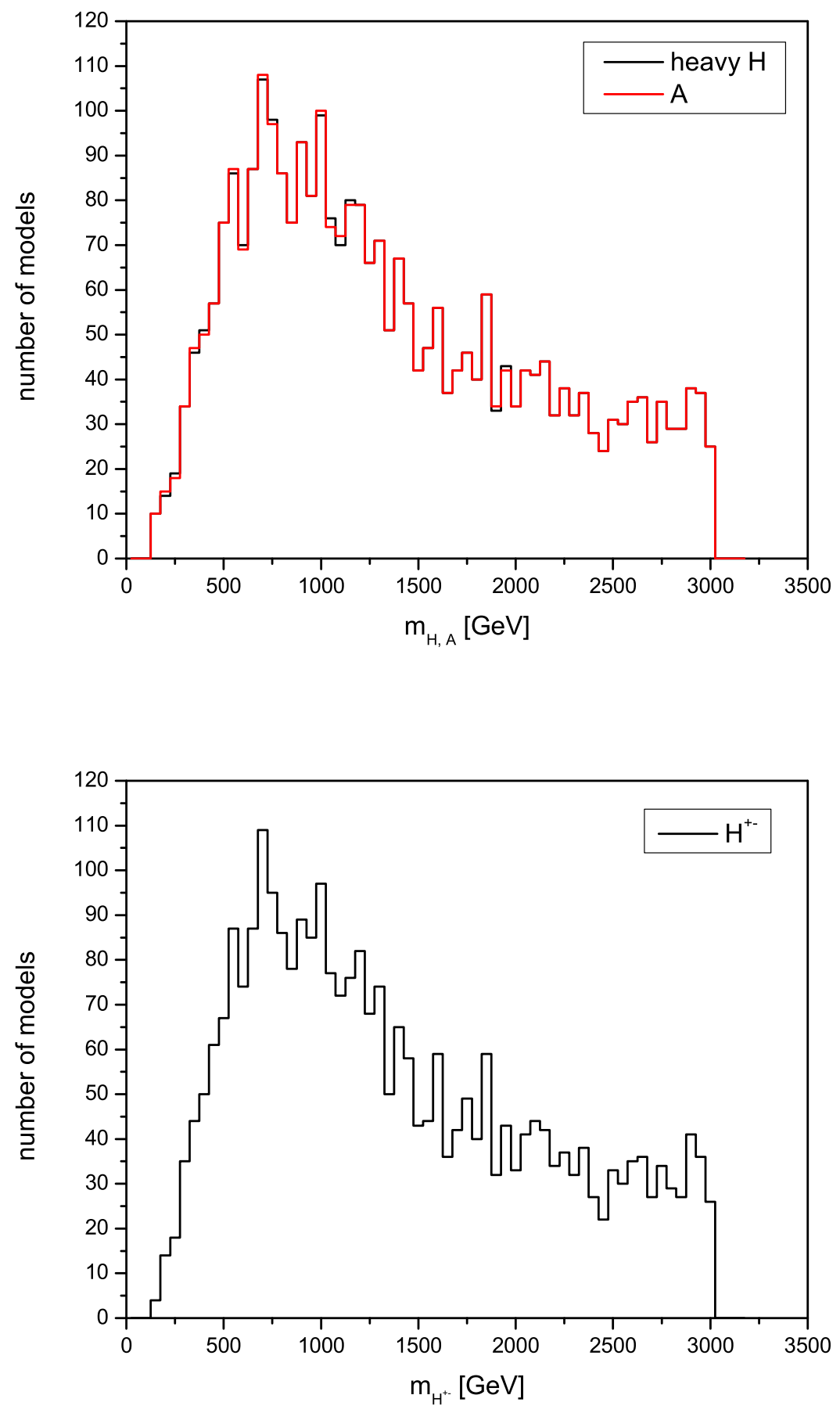

Figure 16: Distribution of heavy and charged Higgs masses for the set of log prior models satisfying all of our constraints. 


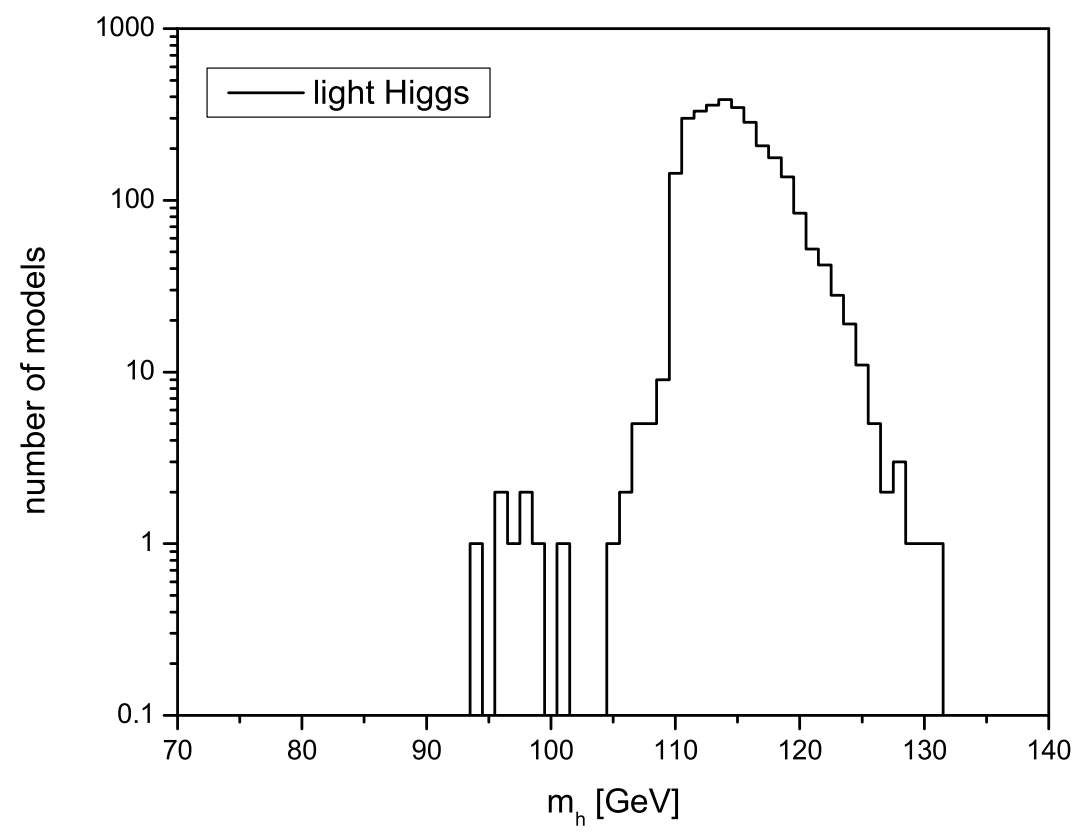

Figure 17: Distribution of the light Higgs mass on a log scale for the set of log prior models satisfying all of our constraints.

in the identity of the nLSP between the log and flat prior scenarios is due to the difference in the statistics of the two sample sizes. Overall, the main distinguishing feature that we observe arises again due to the preference for lighter sparticle masses in the case of log priors and the reduced statistical power of the surviving sample.

The log prior predictions for a number of experimental observables are displayed in Figs. 20 and 21. These are seen to be quite similar to those obtained in the case of flat priors. Some minor differences are: $(i)$ the slight preference for a larger branching fraction for the $b \rightarrow s \gamma$ transition in the case of log priors; (ii) the lack of a bimodal peaking structure for $\Delta(g-2)_{\mu}$ in the $\log$ prior case, which is directly linked to the lighter spartner masses and (iii) though peaked near the same value as in the flat prior sample, the relative number of models in the log prior sample that predict larger values of $\Omega h^{2}$ is found to be somewhat larger. All of these small effects can be traced to the log tilted mass spectra and the corresponding extended mass range. In Fig. 22 we see that the log 

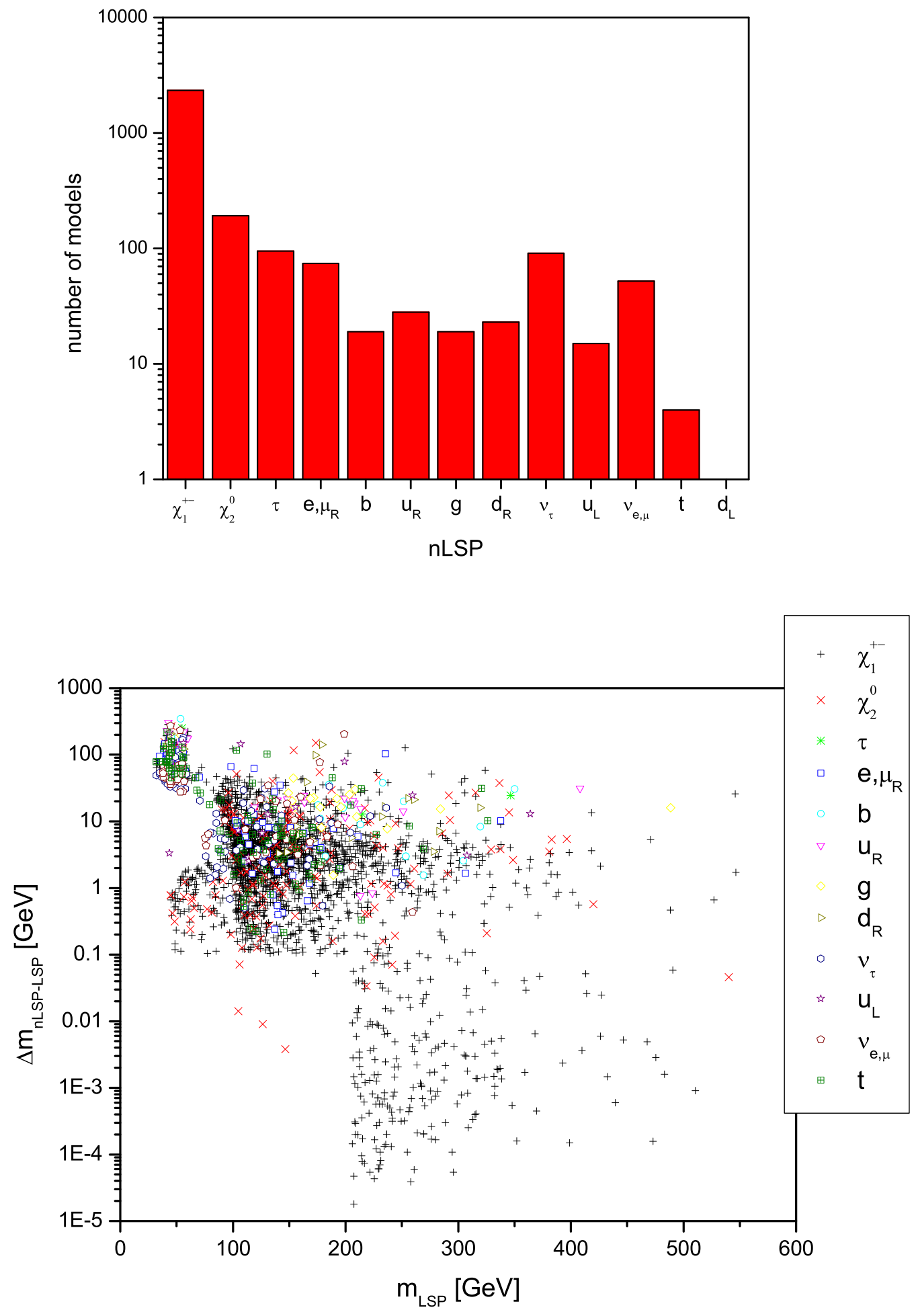

Figure 18: (Top) Identity of the nLSP. (Bottom) nLSP-LSP mass splitting as a function of the LSP mass. Both apply in the case of log priors. 

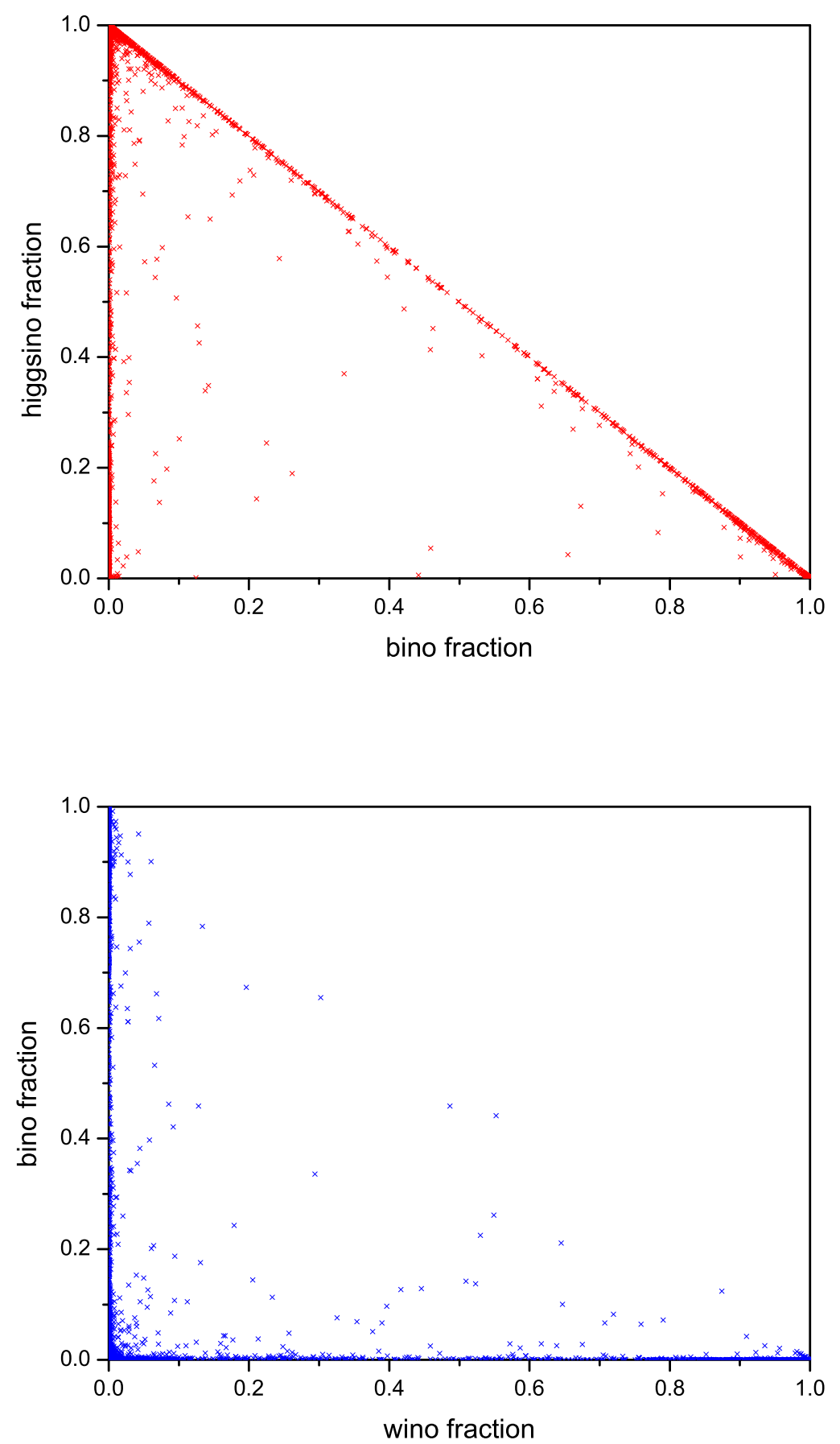

Figure 19: Wino/Higgsino/Bino content of the LSP in the case of log priors. 

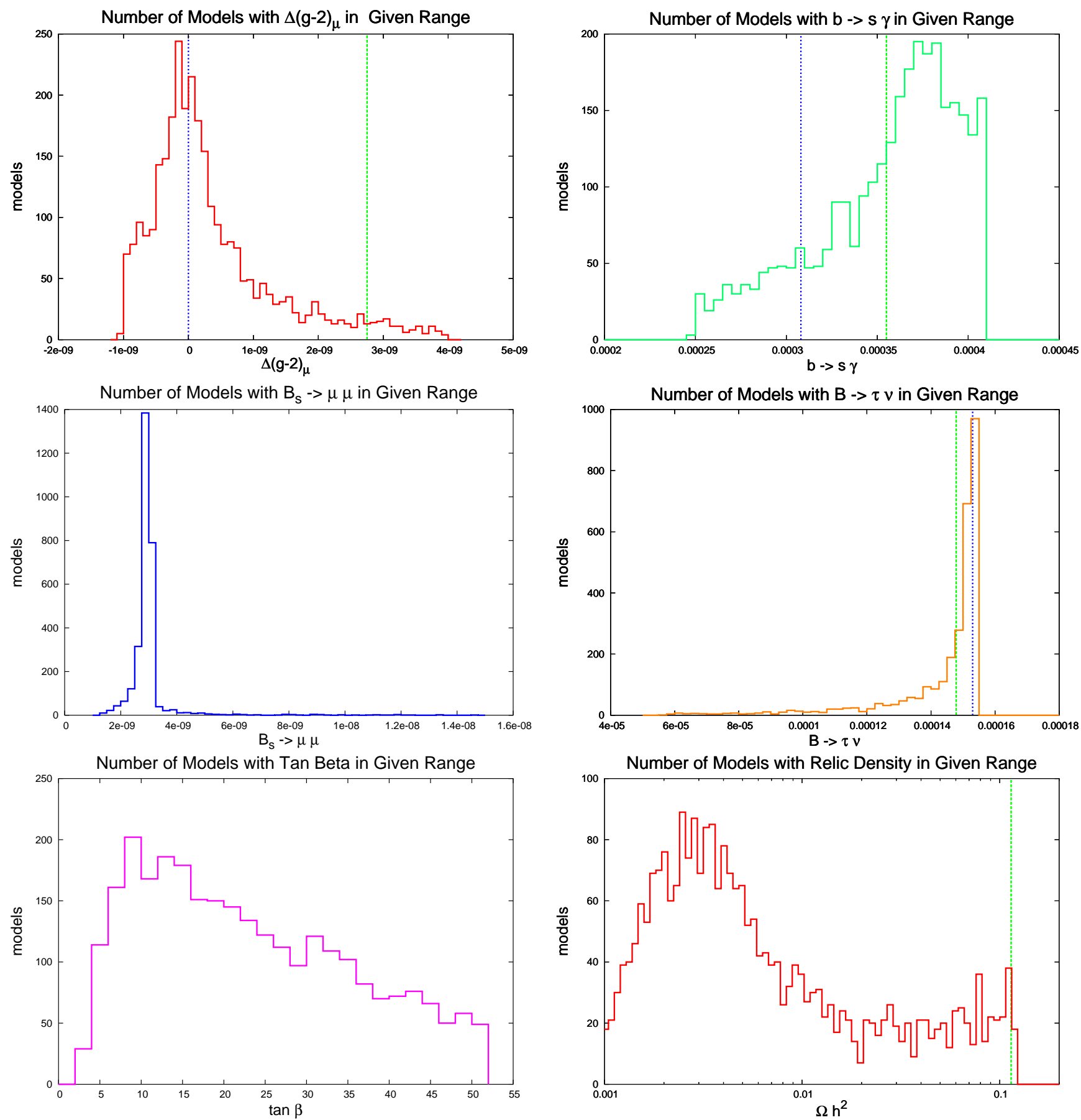

Figure 20: Distributions of predictions for several observables as well as $\tan \beta$ for our model sample subject to the constraints discussed in the text in the case of log priors. The blue and green dashed lines show the SM predictions as well as the current central values obtained by experiment, respectively. 
prior models are perhaps even less fine tuned than those obtained in the flat prior case which is not a surprise since lighter spartner masses are now favored.

From the analysis presented above, we conclude that on the whole it would seem safe to say that the flat and log priors lead to very similar results for almost all of the quantities examined with no significantly large differences.

\section{Discussion}

There are many ways to begin a detailed collider study of the varied signatures arising from the very large set of models that we have generated. Such studies are beyond the scope of the present paper but there are some observations that we can make in addition to those above.

One of the best ways to probe the phenomenological implications of our set of models for the LHC is to examine the mass spectrum beyond the LSP and nLSP. Feldman, Liu and Nath (FLN)[65] have performed a similar study within mSUGRA where they categorize and examine the 22 possible orderings for the 4 lightest new particles in the spectrum. These are rank ordered by the number of times they occur while scanning the parameter space. This mass spectrum ordering is important as it influences the various cascade decay chains for heavy colored SUSY particles produced at the LHC. To get a feeling for our results, we have repeated this analysis using both our flat and log prior model sets. The first question we addressed was how frequently do we obtain a model which can be identified as one of the 22 found by FLN for either set of priors. This is answered in Table 2. Interestingly, we see that one of the FLN orderings, mSP10, does not occur in either of our flat or log prior model sample. Many of the other FLN models are seen to occur rather rarely in our two model sets, whereas the most common FLN models are also common in our two model sets.

The next question we addressed was how many mass patterns do we find for the 4 lightest new particles for both sets of priors and how common are they. This is a generalization of the FLN 

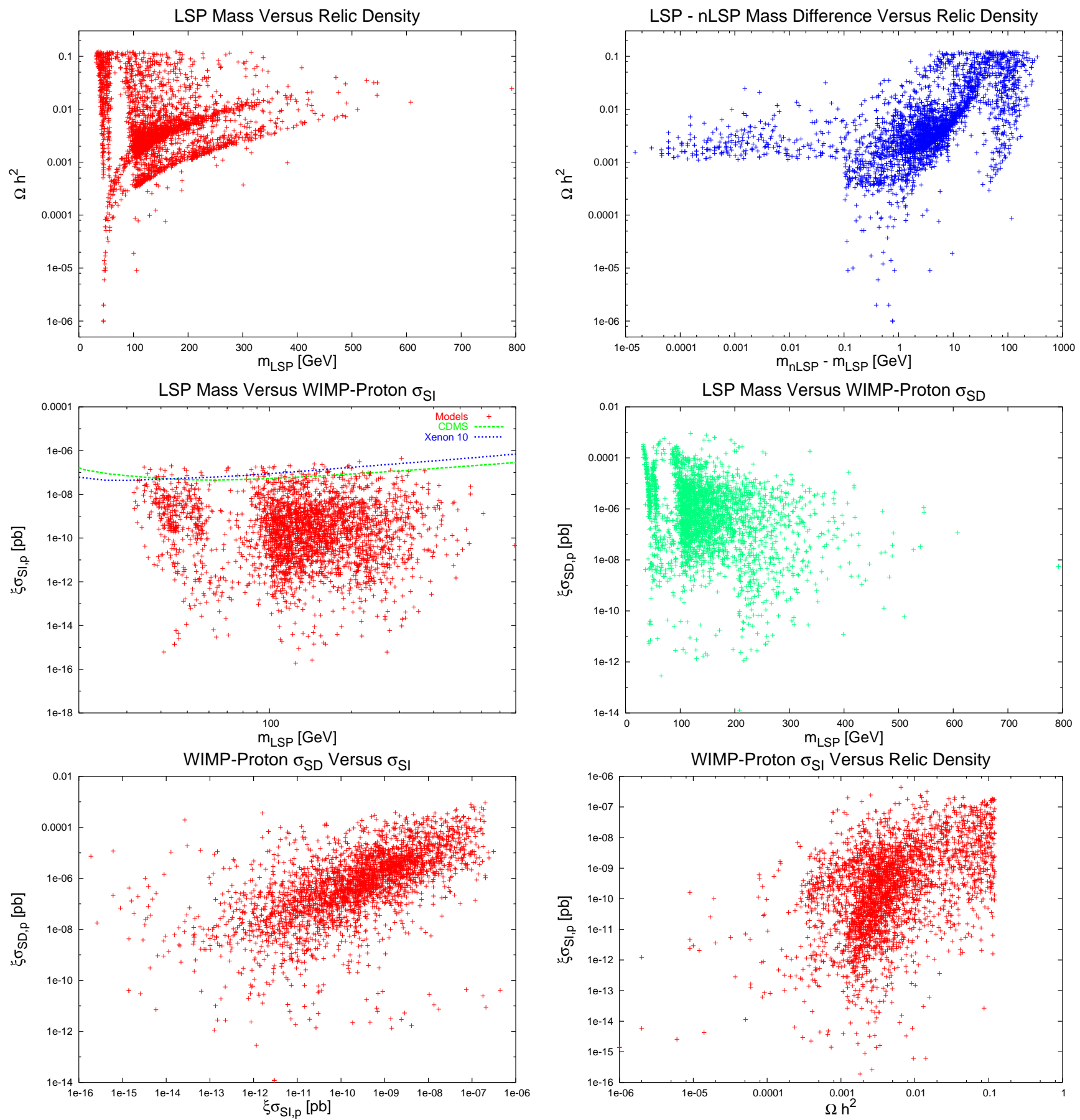

Figure 21: Distributions of relevant dark matter predictions for our model sample in the case of log priors subject to the constraints discussed in the text. All cross sections are in pb. 

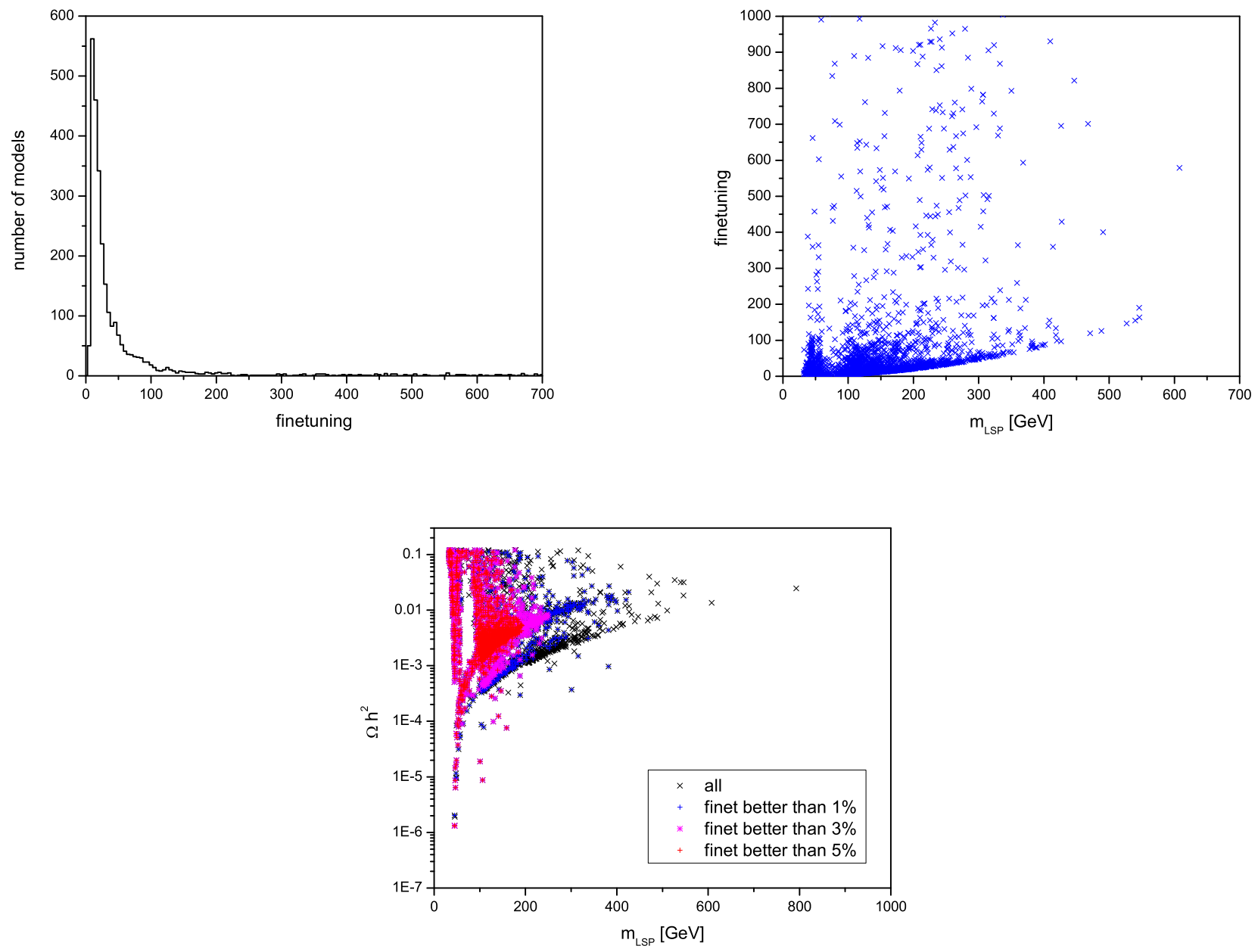

Figure 22: The distribution of fine-tuning $(=\Delta)$ in our set of models with log priors and as a function of the LSP mass. Also shown is the correlation of the amount of fine-tuning with the predicted dark matter density. 


\begin{tabular}{|c|c|c|c|}
\hline \multirow[b]{2}{*}{$\mathrm{mSP}$} & \multirow[b]{2}{*}{ Mass Pattern } & \multicolumn{2}{|c|}{$\%$ of Models } \\
\hline & & Linear Priors & Log Priors \\
\hline mSP1 & $\tilde{\chi}_{1}^{0}<\tilde{\chi}_{1}^{ \pm}<\tilde{\chi}_{2}^{0}<\tilde{\chi}_{3}^{0}$ & 9.82 & 18.59 \\
\hline mSP2 & $\tilde{\chi}_{1}^{0}<\tilde{\chi}_{1}^{ \pm}<\tilde{\chi}_{2}^{0}<A / H$ & 2.08 & 0.68 \\
\hline mSP3 & $\tilde{\chi}_{1}^{0}<\tilde{\chi}_{1}^{ \pm}<\tilde{\chi}_{2}^{0}<\tilde{\tau}_{1}$ & 5.31 & 6.64 \\
\hline $\mathrm{mSP} 4$ & $\tilde{\chi}_{1}^{0}<\tilde{\chi}_{1}^{ \pm}<\tilde{\chi}_{2}^{0}<\tilde{g}$ & 2.96 & 3.73 \\
\hline mSP5 & $\tilde{\chi}_{1}^{0}<\tilde{\tau}_{1}<\tilde{\ell}_{R}<\tilde{\nu}_{\tau}$ & 0.02 & 0.14 \\
\hline mSP6 & $\tilde{\chi}_{1}^{0}<\tilde{\tau}_{1}<\tilde{\chi}_{1}^{ \pm}<\tilde{\chi}_{2}^{0}$ & 0.46 & 1.22 \\
\hline $\mathrm{mSP} 7$ & $\tilde{\chi}_{1}^{0}<\tilde{\tau}_{1}<\tilde{\ell}_{R}<\tilde{\chi}_{1}^{ \pm}$ & 0.02 & 0.03 \\
\hline mSP8 & $\tilde{\chi}_{1}^{0}<\tilde{\tau}_{1}<A \sim H$ & 0.10 & 0 \\
\hline mSP9 & $\tilde{\chi}_{1}^{0}<\tilde{\tau}_{1}<\tilde{\ell}_{R}<A / H$ & 0.01 & 0 \\
\hline mSP10 & $\tilde{\chi}_{1}^{0}<\tilde{\tau}_{1}<\tilde{t}_{1}<\tilde{\ell}_{R}$ & 0 & 0 \\
\hline mSP11 & $\tilde{\chi}_{1}^{0}<\tilde{t}_{1}<\tilde{\chi}_{1}^{ \pm}<\tilde{\chi}_{2}^{0}$ & 0.09 & 0 \\
\hline $\mathrm{mSP} 12$ & $\tilde{\chi}_{1}^{0}<\tilde{t}_{1}<\tilde{\tau}_{1}<\tilde{\chi}_{1}^{ \pm}$ & 0.01 & 0 \\
\hline $\mathrm{mSP} 13$ & $\tilde{\chi}_{1}^{0}<\tilde{t}_{1}<\tilde{\tau}_{1}<\tilde{\ell}_{R}$ & 0.01 & 0 \\
\hline mSP14 & $\tilde{\chi}_{1}^{0}<A \sim H<H^{ \pm}$ & 0.35 & 0.10 \\
\hline mSP15 & $\tilde{\chi}_{1}^{0}<A \sim H<\tilde{\chi}_{1}^{ \pm}$ & 0.08 & 0 \\
\hline mSP16 & $\tilde{\chi}_{1}^{0}<A \sim H<\tilde{\tau}_{1}$ & 0.01 & 0.03 \\
\hline $\mathrm{mSP} 17$ & $\tilde{\chi}_{1}^{0}<\tilde{\tau}_{1}<\tilde{\chi}_{2}^{0}<\tilde{\chi}_{1}^{ \pm}$ & 0.18 & 0.41 \\
\hline $\mathrm{mSP} 18$ & $\tilde{\chi}_{1}^{0}<\tilde{\tau}_{1}<\tilde{\ell}_{R}<\tilde{t}_{1}$ & 0.01 & 0 \\
\hline mSP19 & $\tilde{\chi}_{1}^{0}<\tilde{\tau}_{1}<\tilde{t}_{1}<\tilde{\chi}_{1}^{ \pm}$ & 0.01 & 0 \\
\hline mSP20 & $\tilde{\chi}_{1}^{0}<\tilde{t}_{1}<\tilde{\chi}_{2}^{0}<\tilde{\chi}_{1}^{ \pm}$ & 0.06 & 0 \\
\hline mSP21 & $\tilde{\chi}_{1}^{0}<\tilde{t}_{1}<\tilde{\tau}_{1}<\tilde{\chi}_{2}^{0}$ & 0.01 & 0 \\
\hline mSP22 & $\tilde{\chi}_{1}^{0}<\tilde{\chi}_{2}^{0}<\tilde{\chi}_{1}^{ \pm}<\tilde{g}$ & 0.27 & 0.51 \\
\hline
\end{tabular}

Table 2: Frequency of occurence for the FLN[65] model sequences in our our flat and log prior model samples. 
analysis to the pMSSM. In the case of flat $(\log )$ priors we find 1109(267) different mass patterns for the four lightest sparticles; the difference in these two numbers is largely due to the much greater statistics in the the flat prior case. However, in either scenario this number is many times larger than that found by FLN. This result should not be surprising since we are imposing no constraints on the SUSY breaking mechanism and hence the sparticle mass ordering above the LSP is in general completely arbitrary. Table 3 shows the set of 25 particle mass orderings for the 4 lightest sparticles that occur most frequently amongst our surviving models for both flat and log priors. Note that the sets for the flat and log priors are generically similar but are different in detail. Note that the most frequently occurring FLN orderings are also among the 25 that we obtain for either set of priors. Conversely, however, most of the FLN orderings do not appear amongst our top 25.

In conclusion, we see that the predictions of mSUGRA are greatly extended when examining the pMSSM.

\section{Conclusions}

In this paper we begin a detailed study of the 19 parameter, CP-conserving pMSSM under the assumptions of $(i)$ minimal flavor violation, $(i i)$ the LSP being identified with the lightest neutralino, (iii) only the Yukawa couplings of the third generation are important and (iv) the WMAP measurement of the dark matter relic density is taken only as an upper limit to that arising from the LSP. Carefully subjecting numerous combinations of parameter space points, with mass terms randomly generated assuming either flat or log priors, to a large number of theoretical and experimental constraints we arrive at two sets of models that survive all of the restrictions.

With these two sets of models, we first examined the resulting distributions of expected Higgs and sparticle masses; qualitatively and even semi-quantitatively either choice of prior was found to return quite similar results except for trivially obvious differences due to how the parameters were generated. In both cases, we found some instances where surprisingly light sparticles are still allowed by the data. Next, we studied the identity of the nLSP and its mass splitting with 


\begin{tabular}{|c|c|c|c|}
\hline \multicolumn{2}{|c|}{ Linear Priors } & \multicolumn{2}{|c|}{ Log Priors } \\
\hline Mass Pattern & $\%$ of Models & Mass Pattern & $\%$ of Models \\
\hline$\tilde{\chi}_{1}^{0}<\tilde{\chi}_{1}^{ \pm}<\tilde{\chi}_{2}^{0}<\tilde{\chi}_{3}^{0}$ & 9.82 & $\tilde{\chi}_{1}^{0}<\tilde{\chi}_{1}^{ \pm}<\tilde{\chi}_{2}^{0}<\tilde{\chi}_{3}^{0}$ & 18.59 \\
\hline$\tilde{\chi}_{1}^{0}<\tilde{\chi}_{1}^{ \pm}<\tilde{\chi}_{2}^{0}<\tilde{\ell}_{R}$ & 5.39 & $\tilde{\chi}_{1}^{0}<\tilde{\chi}_{1}^{ \pm}<\tilde{\chi}_{2}^{0}<\tilde{\nu}_{\tau}$ & 7.72 \\
\hline$\tilde{\chi}_{1}^{0}<\tilde{\chi}_{1}^{ \pm}<\tilde{\chi}_{2}^{0}<\tilde{\tau}_{1}$ & 5.31 & $\tilde{\chi}_{1}^{0}<\tilde{\chi}_{1}^{ \pm}<\tilde{\chi}_{2}^{0}<\tilde{\ell}_{R}$ & 6.67 \\
\hline$\tilde{\chi}_{1}^{0}<\tilde{\chi}_{1}^{ \pm}<\tilde{\chi}_{2}^{0}<\tilde{\nu}_{\tau}$ & 5.02 & $\tilde{\chi}_{1}^{0}<\tilde{\chi}_{1}^{ \pm}<\tilde{\chi}_{2}^{0}<\tilde{\tau}_{1}$ & 6.64 \\
\hline$\tilde{\chi}_{1}^{0}<\tilde{\chi}_{1}^{ \pm}<\tilde{\chi}_{2}^{0}<\tilde{b}_{1}$ & 4.89 & $\tilde{\chi}_{1}^{0}<\tilde{\chi}_{1}^{ \pm}<\tilde{\chi}_{2}^{0}<\tilde{d}_{R}$ & 5.18 \\
\hline$\tilde{\chi}_{1}^{0}<\tilde{\chi}_{1}^{ \pm}<\tilde{\chi}_{2}^{0}<\tilde{d}_{R}$ & 4.49 & $\tilde{\chi}_{1}^{0}<\tilde{\chi}_{1}^{ \pm}<\tilde{\chi}_{2}^{0}<\tilde{\nu}_{\ell}$ & 4.50 \\
\hline$\tilde{\chi}_{1}^{0}<\tilde{\chi}_{1}^{ \pm}<\tilde{\chi}_{2}^{0}<\tilde{u}_{R}$ & 3.82 & $\tilde{\chi}_{1}^{0}<\tilde{\chi}_{1}^{ \pm}<\tilde{\chi}_{2}^{0}<\tilde{b}_{1}$ & 3.76 \\
\hline$\tilde{\chi}_{1}^{0}<\tilde{\chi}_{1}^{ \pm}<\tilde{\chi}_{2}^{0}<\tilde{g}$ & 2.96 & $\tilde{\chi}_{1}^{0}<\tilde{\chi}_{1}^{ \pm}<\tilde{\chi}_{2}^{0}<\tilde{g}$ & 3.73 \\
\hline$\tilde{\chi}_{1}^{0}<\tilde{\chi}_{1}^{ \pm}<\tilde{\chi}_{2}^{0}<\tilde{\nu}_{\ell}$ & 2.67 & $\tilde{\chi}_{1}^{0}<\tilde{\chi}_{1}^{ \pm}<\tilde{\chi}_{2}^{0}<\tilde{u}_{R}$ & 2.74 \\
\hline$\tilde{\chi}_{1}^{0}<\tilde{\chi}_{1}^{ \pm}<\tilde{\chi}_{2}^{0}<\tilde{u}_{L}$ & 2.35 & $\tilde{\chi}_{1}^{0}<\tilde{\chi}_{1}^{ \pm}<\tilde{\nu}_{\tau}<\tilde{\tau}_{1}$ & 2.27 \\
\hline$\tilde{\chi}_{1}^{0}<\tilde{\chi}_{1}^{ \pm}<\tilde{\nu}_{\tau}<\tilde{\tau}_{1}$ & 2.19 & $\tilde{\chi}_{1}^{0}<\tilde{\chi}_{2}^{0}<\tilde{\chi}_{1}^{ \pm}<\tilde{\chi}_{3}^{0}$ & 2.24 \\
\hline$\tilde{\chi}_{1}^{0}<\tilde{\chi}_{2}^{0}<\tilde{\chi}_{1}^{ \pm}<\tilde{\chi}_{3}^{0}$ & 2.15 & $\tilde{\chi}_{1}^{0}<\tilde{\chi}_{1}^{ \pm}<\tilde{\ell}_{R}<\tilde{\chi}_{2}^{0}$ & 1.42 \\
\hline$\tilde{\chi}_{1}^{0}<\tilde{\chi}_{1}^{ \pm}<\tilde{\chi}_{2}^{0}<A$ & 2.00 & $\tilde{\chi}_{1}^{0}<\tilde{\chi}_{1}^{ \pm}<\tilde{\chi}_{2}^{0}<\tilde{u}_{L}$ & 1.32 \\
\hline$\tilde{\chi}_{1}^{0}<\tilde{\chi}_{1}^{ \pm}<\tilde{\chi}_{2}^{0}<\tilde{t}_{1}$ & 1.40 & $\tilde{\chi}_{1}^{0}<\tilde{\tau}_{1}<\tilde{\chi}_{1}^{ \pm}<\tilde{\chi}_{2}^{0}$ & 1.22 \\
\hline$\tilde{\chi}_{1}^{0}<\tilde{\chi}_{1}^{ \pm}<\tilde{\nu}_{\ell}<\tilde{\ell}_{L}$ & 1.37 & $\tilde{\chi}_{1}^{0}<\tilde{\chi}_{1}^{ \pm}<\tilde{\tau}_{1}<\tilde{\chi}_{2}^{0}$ & 1.19 \\
\hline$\tilde{\chi}_{1}^{0}<\tilde{\chi}_{1}^{ \pm}<\tilde{\tau}_{1}<\tilde{\chi}_{2}^{0}$ & 1.35 & $\tilde{\chi}_{1}^{0}<\tilde{\chi}_{2}^{0}<\tilde{\chi}_{1}^{ \pm}<\tilde{\nu}_{\tau}$ & 1.15 \\
\hline$\tilde{\chi}_{1}^{0}<\tilde{\chi}_{1}^{ \pm}<\tilde{\ell}_{R}<\tilde{\chi}_{2}^{0}$ & 1.32 & $\tilde{\chi}_{1}^{0}<\tilde{\ell}_{R}<\tilde{\chi}_{1}^{ \pm}<\tilde{\chi}_{2}^{0}$ & 1.05 \\
\hline$A<H<H^{ \pm}<\tilde{\chi}_{1}^{0}$ & 1.24 & $\tilde{\chi}_{1}^{0}<\tilde{\nu}_{\tau}<\tilde{\tau}_{1}<\tilde{\chi}_{1}^{ \pm}$ & 1.02 \\
\hline$\tilde{\chi}_{1}^{0}<\tilde{\chi}_{1}^{ \pm}<\tilde{d}_{R}<\tilde{\chi}_{2}^{0}$ & 1.03 & $\tilde{\chi}_{1}^{0}<\tilde{\chi}_{1}^{ \pm}<\tilde{\nu}_{\ell}<\tilde{\ell}_{L}$ & 0.95 \\
\hline$\tilde{\chi}_{1}^{0}<\tilde{\chi}_{1}^{ \pm}<\tilde{u}_{L}<\tilde{d}_{L}$ & 0.95 & $\tilde{\chi}_{1}^{0}<\tilde{\chi}_{1}^{ \pm}<\tilde{d}_{R}<\tilde{\chi}_{2}^{0}$ & 0.71 \\
\hline$\tilde{\chi}_{1}^{0}<\tilde{\chi}_{1}^{ \pm}<\tilde{b}_{1}<\tilde{\chi}_{2}^{0}$ & 0.89 & $\tilde{\chi}_{1}^{0}<\tilde{\nu}_{\tau}<\tilde{\chi}_{1}^{ \pm}<\tilde{\chi}_{2}^{0}$ & 0.68 \\
\hline$\tilde{\chi}_{1}^{0}<\tilde{\chi}_{1}^{ \pm}<\tilde{u}_{R}<\tilde{\chi}_{2}^{0}$ & 0.84 & $\tilde{\chi}_{1}^{0}<\tilde{\chi}_{1}^{ \pm}<\tilde{\chi}_{2}^{0}<A$ & 0.64 \\
\hline$\tilde{\chi}_{1}^{0}<\tilde{\chi}_{1}^{ \pm}<A<H$ & 0.74 & $\tilde{\chi}_{1}^{0}<\tilde{\chi}_{1}^{ \pm}<\tilde{\nu}_{\tau}<\tilde{\chi}_{2}^{0}$ & 0.61 \\
\hline$\tilde{\chi}_{1}^{0}<\tilde{\chi}_{1}^{ \pm}<\tilde{g}<\tilde{\chi}_{2}^{0}$ & 0.65 & $\tilde{\chi}_{1}^{0}<\tilde{\chi}_{2}^{0}<\tilde{\chi}_{1}^{ \pm}<\tilde{d}_{R}$ & 0.54 \\
\hline$\tilde{\chi}_{1}^{0}<\tilde{\chi}_{1}^{ \pm}<\tilde{\tau}_{1}<\tilde{\nu}_{\tau}$ & 0.51 & $\tilde{\chi}_{1}^{0}<\tilde{\chi}_{1}^{ \pm}<\tilde{\tau}_{1}<\tilde{\nu}_{\tau}$ & 0.54 \\
\hline
\end{tabular}

Table 3: The 25 most common mass orderings for the four lightest sparticles amongst ourmodel set assuming either flat (left) or $\log$ (right) priors. 
the LSP. We found that for either choice of priors, over a dozen sparticles can play the role of the nLSP with more or less the same probabilities in the two model samples. The most common nLSP identities were found to be $\tilde{\chi}_{1}^{ \pm}$and $\tilde{\chi}_{2}^{0}$ with other possibilities being $\tilde{e}_{R}, \tilde{\tau}_{1}$ and $\tilde{t}_{1}$. Less familiar nLSP identities were also found to occur such as the gluino or the first/second generation squarks. We also showed that the mass splitting between these two states, $\Delta m \equiv m_{n L S P}-m_{L S P}$, which is a critical parameter for collider signals and studies, was found to vary over a very large range of six orders of magnitude with values in excess of $\sim 100 \mathrm{GeV}$ and as small as $\sim 100 \mathrm{keV}$ or less. The greatest range was found in the case where the lightest chargino was the nLSP. Small values of $\Delta m$ were found to be quite common particularly in the case of the lightest chargino or the second lightest neutralino being the nLSP. This would imply that searches for long-lived and (essentially) stable particles at the LHC will be of particular importance. In some cases, squarks and gluinos were found to be relatively light and not widely separated in mass from the corresponding LSP. The decays of such states would likely lead to soft jets in the final state which may be difficult to observe above SM backgrounds at the LHC. The mass spectra of the models we obtained were such as to substantially diminish the needed amount of fine tuning in comparison to, e.g., mSUGRA-based scenarios.

Subsequently, we examined the postdictions arising from both model sets for a number of observables such as the branching fraction for $b \rightarrow s \gamma$ and the shift in the value of the $g-2$ of the muon; these were found to be reasonably similar for both cases of priors. Since the predicted dark matter density was allowed to float to any value at or below that obtained by WMAP, we found that the average contribution of the LSP to dark matter peaked at $\sim 4 \%$, with long tails, implying that most of the time some other source of dark matter must typically dominate. However, we do see that there is a reasonable fraction of models where the LSP contribution to the dark matter density is, in fact, quite appreciable in comparison to the WMAP measurement. These results were found to hold for either choice of priors. We then went further and examined the implications of these models for the direct detection of dark matter; we found that the range of possible cross sections is far larger than that obtained in any of the well-known SUSY breaking frameworks, such 
as the cMSSM or mSUGRA. Furthermore these ranges extended into the regions one would have expected to only arise from either the Universal Extra Dimensions or Little Higgs models. This implies that these observables alone cannot be used to discriminate between different Terrascale models.

Clearly the detailed study of the pMSSM models is quite exciting as well as complex; we are just at the initial stages of this analysis and there are many things remaining to be done which we will address in subsequent publications. In addition, we plan to make our models sets public in the near future, in order to encourage a wider set of possible analyses. Hopefully we will not have to wait too long until we actually have real data from the LHC to examine.

\section{Acknowledgments}

The authors would like to thank J. Conley, R. Cotta and D. Maitre for computational aid and S. Kraml and T. Tait for discussions related to this work. They would also like to thank M. Peskin for the suggested title of the paper and J. Baglio for his input during the initial stages of this analysis. CFB thanks the Institut für Physik, Fachbereich Theoretische Physik, Karl-FranzensUniversität Graz and the LPSC Grenoble for hospitality. CFB's work is supported in part by funds provided by the U.S. Department of Energy (D.O.E.) under cooperative research agreement DE-FC02-94ER40818. 


\section{References}

[1] See, for example, G. Bertone, D. Hooper and J. Silk, Phys. Rept. 405, 279 (2005) [arXiv:hep$\mathrm{ph} / 0404175]$.

[2] J. R. Primack, D. Seckel and B. Sadoulet, Ann. Rev. Nucl. Part. Sci. 38, 751 (1988).

[3] G. Kane and S. Watson, arXiv:0807.2244 [hep-ph]; S. Arrenberg, L. Baudis, K. Kong, K. T. Matchev and J. Yoo, arXiv:0805.4210 [hep-ph]; H. Baer and X. Tata, arXiv:0805.1905 [hep-ph]; S. Matsumoto, T. Moroi and K. Tobe, arXiv:0806.3837 [hep-ph].

[4] E. Cremmer, P. Fayet and L. Girardello, Phys. Lett. B 122, 41 (1983).

[5] G. F. Giudice and R. Rattazzi, Phys. Rept. 322, 419 (1999) [arXiv:hep-ph/9801271]; M. Dine, A. E. Nelson, Y. Nir and Y. Shirman, Phys. Rev. D 53, 2658 (1996) [arXiv:hep-ph/9507378].

[6] L. Randall and R. Sundrum, Nucl. Phys. B 557, 79 (1999) [arXiv:hep-th/9810155]; G. F. Giudice, M. A. Luty, H. Murayama and R. Rattazzi, JHEP 9812, 027 (1998) [arXiv:hep$\mathrm{ph} / 9810442]$.

[7] Z. Chacko, M. A. Luty, A. E. Nelson and E. Ponton, JHEP 0001, 003 (2000) [arXiv:hepph/9911323]; D. E. Kaplan, G. D. Kribs and M. Schmaltz, Phys. Rev. D 62, 035010 (2000) [arXiv:hep-ph/9911293].

[8] H. E. Haber and G. L. Kane, Phys. Rept. 117, 75 (1985); S. P. Martin, arXiv:hep-ph/9709356; D. J. H. Chung, L. L. Everett, G. L. Kane, S. F. King, J. D. Lykken and L. T. Wang, Phys. Rept. 407, 1 (2005) [arXiv:hep-ph/0312378]; L. Pape and D. Treille, Rept. Prog. Phys. 69, 2843 (2006); H.K. Dreiner, H.E. Haber, S.P. Martin, "Two-component spinor techniques and Feynman rules for quantum field theory and supersymmetry," SCIPP-08/08, submitted to Phys. Rep.; M. Drees, R. Godbole and P. Roy, Hackensack, USA: World Scientific (2004) 555 p; H. Baer and X. Tata, Cambridge, UK: Univ. Pr. (2006) 537 p. 
[9] For a review, see, G. D’Ambrosio, G. F. Giudice, G. Isidori and A. Strumia, Nucl. Phys. B 645, 155 (2002) [arXiv:hep-ph/0207036].

[10] A. Djouadi, J. L. Kneur and G. Moultaka, Comput. Phys. Commun. 176, 426 (2007) [arXiv:hep-ph/0211331].

[11] A. Freitas, arXiv:0808.0136 [hep-ph].

[12] E. Komatsu et al. [WMAP Collaboration], arXiv:0803.0547 [astro-ph].

[13] W.R. Gilks, S. Richardson, D.J. Spiegelhalter, eds. Markov Chain Monte Carlo in Practice. (Chapman and Hall, 1996).

[14] For original work on this subject, see A.A. Markov. "Rasprostranenie zakona bol'shih chisel na velichiny, zavisyaschie drug ot druga". Izvestiya Fiziko-matematicheskogo obschestva pri Kazanskom universitete, 2-ya seriya, tom 15, pp 135-156, 1906; A.A. Markov. "Extension of the limit theorems of probability theory to a sum of variables connected in a chain". reprinted in Appendix B of: R. Howard. Dynamic Probabilistic Systems, volume 1: Markov Chains. John Wiley and Sons, 1971; Classical Text in Translation: A. A. Markov, An Example of Statistical Investigation of the Text Eugene Onegin Concerning the Connection of Samples in Chains, trans. David Link. Science in Context 19.4 (2006): 591-600. Online:http://journals.cambridge.org/produc

[15] For an overview, see this discussion given in S. Kraml, arXiv:0710.5117 [hep-ph].

[16] C. Amsler et al. [Particle Data Group], Phys. Lett. B 667, 1 (2008). http://www.slac.stanford.edu/xorg/hfag/

[17] P. A. Baikov, K. G. Chetyrkin and J. H. Kuhn, Phys. Rev. Lett. 101, 012002 (2008) [arXiv:0801.1821 [hep-ph]].

[18] LEP Electroweak Working Group, http://www.cern.ch/LEPEWWG. 
[19] [CDF Collaboration and D0 Collaboration], arXiv:0803.1683 [hep-ex].

[20] T. Onogi, talk given at the 34th International Conference on High Energy Physics, Philadelphia, PA, 29 July-5 Aug, 2008.

[21] Heavy Flavor Averaging Group, http://www.slac.stanford.edu/xorg/hfag/.

[22] There have been many such a analyses; a few recent representative examples are: B. C. Allanach, M. J. Dolan and A. M. Weber, JHEP 0808, 105 (2008) [arXiv:0806.1184 [hep-ph]]; S. S. AbdusSalam, arXiv:0809.0284 [hep-ph]; O. Buchmueller et al., arXiv:0808.4128 [hep-ph]; R. Trotta, F. Feroz, M. P. Hobson, L. Roszkowski and R. R. de Austri, arXiv:0809.3792 [hepph]; U. Chattopadhyay and D. Das, arXiv:0809.4065 [hep-ph]; S. Heinemeyer, X. Miao, S. Su and G. Weiglein, arXiv:0805.2359 [hep-ph]; J. R. Ellis, S. Heinemeyer, K. A. Olive, A. M. Weber and G. Weiglein, JHEP 0708, 083 (2007) [arXiv:0706.0652 [hep-ph]]; E. A. Baltz, M. Battaglia, M. E. Peskin and T. Wizansky, Phys. Rev. D 74, 103521 (2006) [arXiv:hep-ph/0602187]; J. Ellis, S. F. King and J. P. Roberts, JHEP 0804, 099 (2008) [arXiv:0711.2741 [hep-ph]].

[23] For a recent review of SUSY Dark Matter candidates, see F. D. Steffen, arXiv:0711.1240 [hep-ph].

[24] See, for example, J. L. Feng, S. Su and F. Takayama, Phys. Rev. D 70, 075019 (2004) [arXiv:hep-ph/0404231] and Phys. Rev. D 70, 063514 (2004) [arXiv:hep-ph/0404198]; M. Fujii and T. Yanagida, Phys. Lett. B 549, 273 (2002) [arXiv:hep-ph/0208191] and Phys. Rev. D 66, 123515 (2002) [arXiv:hep-ph/0207339]; J. R. Ellis, K. A. Olive, Y. Santoso and V. C. Spanos, Phys. Lett. B 588, 7 (2004) [arXiv:hep-ph/0312262]; L. Roszkowski, R. Ruiz de Austri and K. Y. Choi, JHEP 0508, 080 (2005) [arXiv:hep-ph/0408227].

[25] G. Belanger, F. Boudjema, A. Pukhov and A. Semenov, Comput. Phys. Commun. 177 (2007) 894; arXiv:0803.2360 [hep-ph]; Comput. Phys. Commun. 149, 103 (2002) [arXiv:hep- 
ph/0112278]; Comput. Phys. Commun. 174, 577 (2006) [arXiv:hep-ph/0405253]; Comput. Phys. Commun. 176, 367 (2007) [arXiv:hep-ph/0607059] and arXiv:0803.2360 [hep-ph].

[26] M. Misiak et al., Phys. Rev. Lett. 98, 022002 (2007) [arXiv:hep-ph/0609232].

[27] T. Becher and M. Neubert, Phys. Rev. Lett. 98, 022003 (2007) [arXiv:hep-ph/0610067].

[28] The preliminary combined CDF and D0 limit was present by D. Toback, talk given at Physics for LHC-2008, Split, Croatia, 29 Sept.-4 Oct. 2008. See also T. Aaltonen et al. [CDF Collaboration], Phys. Rev. Lett. 100, 101802 (2008) [arXiv:0712.1708 [hep-ex]].

[29] G. W. Bennett et al. [Muon G-2 Collaboration], Phys. Rev. D 73, 072003 (2006) [arXiv:hepex/0602035].

[30] M. Passera, W. J. Marciano and A. Sirlin, arXiv:0809.4062 [hep-ph].

[31] E. De Rafael, arXiv:0809.3085 [hep-ph].

[32] P. Chang, K. Hara and T. Hurth, talks given at the 34th International Conference on High Energy Physics, Philadelphia, PA, 29 July-5 Aug, 2008.

[33] G. Isidori and P. Paradisi, Phys. Lett. B 639, 499 (2006) [arXiv:hep-ph/0605012].

[34] D. Eriksson, F. Mahmoudi and O. Stal, arXiv:0808.3551 [hep-ph].

[35] For an introduction, see, J. S. Hagelin, S. Kelley and T. Tanaka, Nucl. Phys. B 415, 293 (1994); F. Gabbiani, E. Gabrielli, A. Masiero and L. Silvestrini, Nucl. Phys. B 477, 321 (1996) [arXiv:hep-ph/9604387].

[36] For recent overviews see G. Gelmini, talk given at the 34th International Conference on High Energy Physics, Philadelphia, PA, 29 July-5 Aug, 2008 and U. Oberlack, talk given at COSMO '08, Madison, WI, Aug. 2008; G. Gerbier, talk given at SUSY 008, Seoul, Korea, June 2008. See also J. D. Vergados, Lect. Notes Phys. 720, 69 (2007) [arXiv:hep-ph/0601064]. 
[37] U. Oberlack [XENON Collaboration], J. Phys. Conf. Ser. 110, 062020 (2008).

[38] Z. Ahmed et al. [CDMS Collaboration], arXiv:0802.3530 [astro-ph].

[39] M. Bravin et al. [CRESST-Collaboration], Astropart. Phys. 12, 107 (1999) [arXiv:hepex/9904005].

[40] R. Bernabei et al. [DAMA Collaboration], Phys. Lett. B 480, 23 (2000).

[41] G. Benelli, "Search for stable and long lived heavy charged particles in electron positron collisions at center of mass energies from $130-\mathrm{GeV}$ to $209-\mathrm{GeV}$ with the OPAL detector at LEP," UMI-31-09638, 2003. 126pp.

[42] A. Djouadi, M. M. Muhlleitner and M. Spira, Acta Phys. Polon. B 38, 635 (2007) [arXiv:hepph/0609292]; http://lappweb.in2p3.fr/ muehlleitner/SUSY-HIT/ .

[43] R. Barate et al. [ALEPH Collaboration], Phys. Lett. B 469, 303 (1999).

[44] A. C. Kraan, arXiv:hep-ex/0305051.

[45] LEP SUSY Working Group, http://lepsusy.web.cern.ch/lepsusy/ .

[46] For a review, see, for example, S. Heinemeyer, W. Hollik and G. Weiglein, Phys. Rept. 425, 265 (2006) [arXiv:hep-ph/0412214].

[47] LEP Higgs Working Group, http://lephiggs.web.cern.ch/LEPHIGGS/www/Welcome.html .

[48] V. M. Abazov et al. [D0 Collaboration], Phys. Lett. B 660, 449 (2008) [arXiv:0712.3805 [hep$\mathrm{ex}]]$.

[49] W. Beenakker, R. Hopker and M. Spira, arXiv:hep-ph/9611232. See also, http://www.ph.ed.ac.uk/ tplehn/prospino/ ¡http://www.ph.ed.ac.uk/The NLO squark and gluino cross section are given in W. Beenakker, R. Hopker, M. Spira and P. M. Zerwas, Nucl. Phys. B 492, 51 (1997) [arXiv:hep-ph/9610490]. 
[50] T. Sjostrand, S. Mrenna and P. Skands, JHEP 0605, 026 (2006) [arXiv:hep-ph/0603175].

[51] J. Conway, PGS4, Pretty Good detector Simulation, http://www.physics.ucdavis.edu/ conway/research/software/pgs/pgs.html .

[52] G. J. Feldman and R. D. Cousins, Phys. Rev. D 57, 3873 (1998) [arXiv:physics/9711021].

[53] T. Aaltonen et al. [CDF Collaboration], arXiv:0808.2446 [hep-ex].

[54] A. Duperrin [CDF Collaboration and D0 Collaboration], arXiv:0710.4265 [hep-ex]. For a recent update, see S. Lowette, talk given at the 34th International Conference on High Energy Physics, Philadelphia, PA, 29 July-5 Aug, 2008. For the latest overview, see Tevatron New Phenomena and Higgs Working Group, http://tevnphwg.fnal.gov/ .

[55] F. Abe et al. [CDF Collaboration], Phys. Rev. D 46, 1889 (1992); CDF Note 8701.

[56] V. M. Abazov et al. [D0 Collaboration], arXiv:0809.4472 [hep-ex].

[57] T. Aaltonen et al. [CDF Collaboration], Phys. Rev. Lett. 101, 071802 (2008) [arXiv:0802.3887 [hep-ex]]; CDF Note CDF/PUB/TOP/PUBLIC/9343; D0 Note 5598-CONF; CDF Note 9332; V. M. Abazov et al. [D0 Collaboration], Phys. Rev. Lett. 97, 171806 (2006) [arXiv:hepex/0608013]; V. Buescher et al. [CDF and DO Collaborations], arXiv:hep-ex/0504004; V. M. Abazov et al. [D0 Collaboration], Phys. Lett. B 665, 1 (2008) [arXiv:0803.2263 [hepex]]; T. Aaltonen et al. [CDF Collaboration], Phys. Rev. D 76, 072010 (2007) [arXiv:0707.2567 [hep-ex]].

[58] J. Alwall, M. P. Le, M. Lisanti and J. G. Wacker, arXiv:0809.3264 [hep-ph] and arXiv:0803.0019 [hep-ph].

[59] N. Arkani-Hamed and S. Dimopoulos, JHEP 0506, 073 (2005) [arXiv:hep-th/0405159]; G. F. Giudice and A. Romanino, Nucl. Phys. B 699, 65 (2004) [Erratum-ibid. B 706, 65 (2005)] [arXiv:hep-ph/0406088]. 
[60] N. Arkani-Hamed, G. L. Kane, J. Thaler and L. T. Wang, JHEP 0608, 070 (2006) [arXiv:hep$\mathrm{ph} / 0512190]$.

[61] C. F. Berger, J. S. Gainer, J. L. Hewett, B. Lillie and T. G. Rizzo, arXiv:0712.2965 [hep-ph] and arXiv:0711.1374 [hep-ph].

[62] We would like to thank the authors of this code for communications on this point and note that they have recently corrected this feature in their code.

[63] V. Barger, W. Y. Keung and G. Shaughnessy, arXiv:0806.1962 [hep-ph].

[64] R. Barbieri and G. F. Giudice, Nucl. Phys. B 306, 63 (1988). We follow the analysis as presented in J. Kasahara, K. Freese and P. Gondolo, arXiv:0805.0999 [hep-ph].

[65] D. Feldman, Z. Liu and P. Nath, Phys. Rev. Lett. 99, 251802 (2007) [Erratum-ibid. 100, 069902 (2008)] [arXiv:0707.1873 [hep-ph]] and JHEP 0804, 054 (2008) [arXiv:0802.4085 [hep$\mathrm{ph}]$. 